\title{
Detailed study of the $\Lambda_{b} \rightarrow \Lambda \ell^{+} \ell^{-}$decays in the standard model
}

\author{
Srimoy Bhattacharya, ${ }^{1, *}$ Soumitra Nandi, ${ }^{2, \dagger}$ Sunando Kumar Patra $\oplus^{2, *}$ and Ria Sain $\circledast^{1, \S}$ \\ ${ }^{1}$ The Institute of Mathematical Sciences, Taramani, Chennai 600113, India \\ ${ }^{2}$ Indian Institute of Technology, North Guwahati, Guwahati 781039, Assam, India
}

(Received 24 December 2019; accepted 11 April 2020; published 30 April 2020)

\begin{abstract}
Based on the standard model (SM) of particle physics, we study the decays $\Lambda_{b} \rightarrow \Lambda \ell^{+} \ell^{-}$in light of the available inputs from lattice simulations and data from LHCb. We fit the form factors of this decay mode using the available theoretical and experimental inputs after defining different fit scenarios and checking for consistency. The theory inputs include the relations between the form factors in heavy quark effective theory and soft collinear effective theory at the end points of the dilepton invariant mass squared $q^{2}$. Utilizing the fit results, we predict a few observables related to this mode. We also predict the observable $R_{\Lambda}=\operatorname{Br}\left(\Lambda_{b} \rightarrow \Lambda \ell_{i}^{+} \ell_{i}^{-}\right) / \operatorname{Br}\left(\Lambda_{b} \rightarrow \Lambda \ell_{j}^{+} \ell_{j}^{-}\right)$, where $\ell_{i}$ and $\ell_{j}$ are charged leptons of different generations $(i \neq j)$. At the moment, we do not observe noticeable differences in the extracted values of the observables in fully data-driven and SM-like fit scenarios.
\end{abstract}

DOI: 10.1103/PhysRevD.101.073006

\section{INTRODUCTION}

The flavor-changing neutral current (FCNC) $b \rightarrow s$ transitions play an important role in indirect searches for new physics (NP). In recent years, special attention has been given to the semileptonic $b \rightarrow s \ell^{+} \ell^{-}$decays, such as $B \rightarrow K^{(*)} \mu^{+} \mu^{-}$and $B_{s} \rightarrow \phi \mu^{+} \mu^{-}$. Precise measurements of various angular observables are now available. On top of this, measurements are done on ratios like $R_{K}^{(*)}=$ $\left(B \rightarrow K^{(*)} \mu^{+} \mu^{-}\right) /\left(B \rightarrow K^{(*)} e^{+} e^{-}\right)$. The results show some degree of discrepancy with their respective standard model (SM) predictions $[1,2]$. For an update, see the most recent analysis [3] and references therein.

The observed differences could be due to either some new interactions beyond the SM (BSM), our poor understanding of the hadronic uncertainties, or our inability to correctly analyze the experimental data. In spite of the obvious lure and consequent multitude of possible explanations of these deviations from BSM effects, it is crucial to investigate and refine the existing theoretical description of the large hadronic effects in the rare $b \rightarrow s$ transitions. The study of various other similar decay modes can provide complementary phenomenological information compared

\footnotetext{
*bhattacharyasrimoy@gmail.com

†soumitra.nandi@iitg.ac.in

*sunando.patra@gmail.com

§riasain@imsc.res.in
}

Published by the American Physical Society under the terms of the Creative Commons Attribution 4.0 International license. Further distribution of this work must maintain attribution to the author(s) and the published article's title, journal citation, and DOI. Funded by SCOAP ${ }^{3}$. to the above-mentioned well-analyzed mesonic decays, which can be useful to improve our understanding of the nature of the anomalies seen in the $B$-meson decays. Moreover, any BSM physics altering the results for these modes should affect and be constrained by other $b \rightarrow s$ transitions.

Among all such processes, the baryonic decay mode $\Lambda_{b} \rightarrow \Lambda \ell^{+} \ell^{-}$is of considerable interest for several reasons:

(1) In the ground state, $\Lambda_{b}$ is the combination of a heavy quark and a light diquark system. The light quarks are in a spin-zero state, which leads to a simpler theoretical description of the semileptonic decays of $\Lambda_{b}$ baryons compared to the corresponding meson decays.

(2) As the $\Lambda_{b}$ baryon has nonzero spin, this process (unlike the mesonic decays) has the potential to improve our limited understanding of the helicity structure of the underlying Hamiltonian [4].

(3) Just like the $B \rightarrow K^{(*)} \ell^{+} \ell^{-}$processes, the polarization of the $\Lambda$ baryon, preserved in the $\Lambda \rightarrow p \pi^{-}$ decay, provides a plethora of angular observables, with the potential to disentangle the contributions from individual operators in the $b \rightarrow s \ell^{+} \ell^{-}$effective Hamiltonian [5-11].

(4) If we consider an unpolarized $\Lambda_{b}$ baryon, then the number of angular observables is restricted to ten. However, if we produce a polarized $\Lambda_{b}$ then the number of angular observables will increase from $10 \rightarrow 34$ [12]. Thus, there will be even more opportunities for testing NP.

In the factorization limit, ten independent form factors are needed to describe the $\Lambda_{b} \rightarrow \Lambda \ell^{+} \ell^{-}$decays. There are 
"nonfactorizable" effects too, which cannot be described in terms of $\Lambda_{b} \rightarrow \Lambda$ form factors. These corrections are not yet available in the literature, and hence we ignore these effects in our analysis. The above-mentioned ten form factors are the major sources of uncertainties in the description of various observables in these decays. Different QCD-based approaches are available in the literature to describe the $q^{2}$ distributions of these form factors (see Refs. [13-16] and references therein for details). The SM predictions, based on the lattice-based analysis given in Ref. [17], have large errors. In addition to the uncertainty in the form factors, some angular observables of $\Lambda_{b}$ decays are plagued by the dependence of the detection efficiencies on the production polarization $\left(P_{\Lambda_{b}}\right)$. The most recent measurement of $P_{\Lambda_{b}}$ by LHCb [18] is quite imprecise $\left(P_{\Lambda_{b}}=0.06 \pm 0.07 \pm 0.02\right)$, and the effect of nonzero polarization has been taken into account as a systematic uncertainty in Ref. [19]. The availability of these measurements, though imprecise for now, gives us a way to make a data-driven estimation of the correlation between $P_{\Lambda_{b}}$ and the form-factor parameters.

Experimental data are available on the decay rate distributions in different $q^{2}$ (the momentum transfer to leptons) bins [19-21]. Moreover, LHCb has very recently measured various angular observables associated with the above decay [22]. Most of the available data have large errors, and it would be premature to assume the presence of new physics and constrain them using this data. Before jumping the gun, it is important and useful to understand the trend of the available data/inputs.

Our main objective in this analysis is to test whether or not all of the available inputs (for example, experimental data, lattice data, and other theory inputs from the QCD modeling of the form factors) for the form factors in $\Lambda_{b} \rightarrow$ $\Lambda \ell^{+} \ell^{-}$decays are consistent with each other. Looking for inconsistencies will help us to improve our understanding of the underlying physics. There exists a number of relations between the form factors of the above-mentioned decay modes in the heavy quark effective theory (HQET) and soft collinear effective theory (SCET), at the end points of their $q^{2}$ distributions $[15,23]$. It will be interesting to see whether the available data and inputs from lattice simulations support these expectations. On the other hand, using these HQET and SCET relations as inputs while extracting the $q^{2}$ distributions of the form factors may help to reduce the uncertainties of the extracted values of the $\Lambda_{b} \rightarrow \Lambda \ell^{+} \ell^{-}$observables.

We analyze the available inputs after creating different fit scenarios with variable inputs (as discussed above) extract the form factors in all of the scenarios, and compare them for consistency. We also predict the branching fraction $\operatorname{Br}\left(\Lambda_{b} \rightarrow \Lambda \ell \ell\right)$, the $q^{2}$ distributions of the branching fractions, the forward-backward asymmetry $A_{\mathrm{FB}}\left(q^{2}\right)$, and the longitudinal polarization asymmetry $f_{L}\left(q^{2}\right)$ using these form factors. Similar to the observables $R_{K^{(*)}}$, we define the observables
$R_{\Lambda}^{\ell_{i} / \ell_{j}}=\left(\operatorname{Br}\left(\Lambda_{b} \rightarrow \Lambda \ell_{i}^{+} \ell_{i}^{-}\right)\right) /\left(\operatorname{Br}\left(\Lambda_{b} \rightarrow \Lambda \ell_{j}^{+} \ell_{j}^{-}\right)\right)$, where $\ell_{i}$ and $\ell_{j}$ are charged leptons of different generations $(i \neq j)$, and give predictions for these observables using our fit results.

\section{FORMALISM}

\section{A. Angular distribution}

The differential decay rate for the $\Lambda_{b} \rightarrow \Lambda \ell^{+} \ell^{-}$decay can be expressed in terms of generalized helicity amplitudes and five variables: the angle $\theta$ between the direction of the $\Lambda$ baryon and the normal vector $\hat{n}$ in the $\Lambda_{b}$ rest frame, two sets of helicity angles describing the decays of the $\Lambda$ baryon $\left(\theta_{b}, \phi_{b}\right)$ and the dilepton system $\left(\theta_{l}, \phi_{l}\right)$, and the invariant mass squared of dilepton $q^{2}$, as given in Eq. (1). For transverse production, the polarization $\hat{n}$ is chosen to be $\hat{p}_{\Lambda_{b}} \times \hat{p}_{\text {beam }}$. The helicity angles are then defined with respect to this normal vector through the coordinate systems $\left(\hat{x}_{\Lambda}, \hat{y}_{\Lambda}, \hat{z}_{\Lambda}\right)$ and $\left(\hat{x}_{\ell \bar{\ell}}, \hat{y}_{\ell \bar{\ell}}, \hat{z}_{\ell \bar{\ell}}\right)$. The $\hat{z}$ axis points in the direction of the $\Lambda$ /dilepton system in the $\Lambda_{b}$ rest frame. The angle between the two decay planes in the $\Lambda_{b}$ rest frame is $\chi=\phi_{l}+\phi_{b}$. The angles $\theta_{l}, \theta_{b}$, and $\chi$ are sufficient to parametrize the angular distribution of the decay in the case of zero production polarization [12]:

$$
\begin{aligned}
\frac{\mathrm{d}^{6} \Gamma}{\mathrm{d} q^{2} \mathrm{~d} \vec{\Omega}} \propto & \sum_{\substack{\lambda_{1}, \lambda_{2}, \lambda_{p}, \lambda_{\ell \ell}, \lambda^{\prime} l_{\ell}^{\prime} \\
J, J^{\prime}, m, m^{\prime}, \lambda_{\Lambda}, \lambda_{\Lambda}^{\prime}}}\left((-1)^{J+J^{\prime}} \rho_{\lambda_{\Lambda}-\lambda_{\ell \ell}, \lambda_{\Lambda}^{\prime}-\lambda_{\ell \ell}^{\prime}}(\theta)\right. \\
& \times H_{\lambda_{\Lambda}, \lambda_{\ell \ell}}^{m, J}\left(q^{2}\right) H_{\lambda_{\Lambda}^{\prime}, \lambda_{\ell \ell}^{\prime}}^{\dagger m^{\prime}, J^{\prime}}\left(q^{2}\right) h_{\lambda_{1}, \lambda_{2}}^{m, J}\left(q^{2}\right) h_{\lambda_{1}, \lambda_{2}}^{\dagger m^{\prime}, J^{\prime}}\left(q^{2}\right) \\
& \times D_{\lambda_{\ell \ell}, \lambda_{1}-\lambda_{2}}^{J}\left(\phi_{l}, \theta_{l},-\phi_{l}\right) \\
& \times D_{\lambda_{\ell,}, \lambda_{1}-\lambda_{2}}^{J^{\prime}}\left(\phi_{l}, \theta_{l},-\phi_{l}\right) h_{\lambda_{p}, 0}^{\Lambda} h_{\lambda_{p} 0}^{\dagger \Lambda} \\
& \left.\times D_{\lambda_{\Lambda}, \lambda_{p}}^{1 / 2}\left(\phi_{b}, \theta_{b},-\phi_{b}\right) D_{\lambda_{\Lambda}^{\prime}, \lambda_{p}}^{1 / 2}\left(\phi_{b}, \theta_{b},-\phi_{b}\right)\right),
\end{aligned}
$$

where $\vec{\Omega}$ depends on five angles, $\vec{\Omega} \equiv \vec{\Omega}\left(\theta_{l}, \phi_{l}, \theta_{b}, \phi_{b}, \theta\right)$.

The $D^{j}$ functions are the Wigner $D$ matrices, which are unitary square matrices of $(2 j+1)$ dimensions. The factor $(-1)^{J+J^{\prime}}$ comes from the structure of the Minkowski metric tensor. The decay distribution contains three sets of helicity amplitudes:

(1) $H_{\lambda_{\mathrm{A}}, \lambda_{\ell \ell}}^{m, J}\left(q^{2}\right)$ defines the decay of the $\Lambda_{\mathrm{b}}$ baryon into a $\Lambda$ baryon with helicity $\lambda_{\Lambda}$ and a dilepton pair with helicity $\lambda_{\ell \ell}$.

(2) $h_{\lambda_{1}, \lambda_{2}}^{m, J}$ describes the decay of the dilepton system to leptons with helicities $\lambda_{1}$ and $\lambda_{2}$.

(3) $h_{\lambda_{p}, 0}^{\Lambda}$ denotes the decay $\Lambda \rightarrow p \pi$ to a proton with helicity $\lambda_{p}$.

The index $J$, which stands for the spin of the dilepton system, can be either zero or one. When $J=0, \lambda_{\ell \ell}=0$, and when $J=1, \lambda_{\ell \ell}$ can be $-1,0,+1$. The helicity labels $\lambda_{p}, \lambda_{\Lambda}, \lambda_{1}$, and $\lambda_{2}$ can take the values $\pm 1 / 2$. From the angular momentum conservation in the $\Lambda_{\mathrm{b}}$ decay we get 
$\left|\lambda_{\Lambda}-\lambda_{\ell \ell}\right|=1 / 2$. The remaining index, $m(=V, A)$, indicates the decay of the dilepton system by either a vector or axial-vector current. The polarization of the parent baryon is described by the density matrix $\rho_{\lambda_{\Lambda}-\lambda_{\ell \ell}, \lambda_{\Lambda}^{\prime}-\lambda_{\ell \ell}^{\prime}}$, which is defined as

$$
\rho_{\lambda, \lambda^{\prime}}=\frac{1}{2}\left(\begin{array}{cc}
1+P_{\Lambda_{\mathrm{b}}} \cos \theta & P_{\Lambda_{\mathrm{b}}} \sin \theta \\
P_{\Lambda_{\mathrm{b}}} \sin \theta & 1-P_{\Lambda_{\mathrm{b}}} \cos \theta
\end{array}\right),
$$

where $P_{\Lambda_{\mathrm{b}}}$ is the polarization of the parent baryon $\Lambda_{\mathrm{b}}$ which we fit along with the other parameters in our analysis. For more details on the angular distributions, please see Refs. [24,25].

\section{B. Form factors}

The helicity amplitudes $H_{\lambda_{\Lambda}, \lambda_{\ell \ell}}^{m, J}\left(q^{2}\right)$ can be expressed in terms of ten form factors. In this paper, we use the helicitybased definition of the form factors from Ref. [15], given in the Appendix A.

Following the parametrization of Ref. [17], each one of these ten form factors can be parametrized in terms of independent parameters $a_{k}^{f_{i}}$ as follows:

$$
f_{i}\left(q^{2}\right)=\frac{1}{1-q^{2} /\left(m_{\text {pole }}^{f_{i}}\right)^{2}} \sum_{k=0}^{N} a_{k}^{f_{i}}\left[z\left(q^{2}\right)\right]^{k} .
$$

Here, $z\left(q^{2}\right)$ is defined as

$$
z\left(q^{2}\right)=\frac{\sqrt{t_{+}-q^{2}}-\sqrt{t_{+}-t_{0}}}{\sqrt{t_{+}-q^{2}}+\sqrt{t_{+}-t_{0}}},
$$

where $t_{0}=\left(m_{\Lambda_{b}}-m_{\Lambda}\right)^{2}$ and $t_{+}=\left(m_{B}+m_{K}\right)^{2}$. The choice of the truncation order $N$ of the $z$ expansion in Eq. (3) determines the number of independent parameters in our fit. The series in Eq. (3) can be truncated at different values of $N$, and in this paper we present our analysis for both $N=1$ and $N=2$. At the present accuracy level, it is hard to do the analysis with $N>2$.

The helicity form factors satisfy the kinematic constraints at the end point: ${ }^{1}$

$$
\begin{gathered}
f_{0}(0)=f_{+}(0), \\
g_{0}(0)=g_{+}(0), \\
g_{\perp}\left(q_{\max }^{2}\right)=g_{+}\left(q_{\max }^{2}\right), \\
\tilde{h}_{\perp}\left(q_{\max }^{2}\right)=\tilde{h}_{+}\left(q_{\max }^{2}\right) .
\end{gathered}
$$

\footnotetext{
${ }^{1}$ In the presence of nonfactorizable corrections these relations may not be exact, and there will be additional corrections. Keeping in mind the present level of accuracy, in this analysis we ignore those corrections.
}

Implementing the constraints (5c) and (5d) is tantamount to setting $a_{0}^{g_{\perp}}=a_{0}^{g_{+}}$and $a_{0}^{\tilde{h}_{\perp}}=a_{0}^{\tilde{h}_{+}}$, respectively, in our analysis. On the other hand, using Eqs. (5a) and (5b), we write $a_{1}^{f_{+}}$and $a_{1}^{g_{+}}$in terms of the other form factor parameters. Throughout our analysis we use these relations.

In the HQET and SCET, there are additional relations between the form factors at the end points of the $q^{2}$ distributions. In HQET, we have the following approximate relations between the form factors for small recoil:

$$
\begin{aligned}
f_{0}\left(q_{\max }^{2}\right) & \simeq g_{+}\left(q_{\max }^{2}\right) \simeq g_{\perp}\left(q_{\max }^{2}\right) \\
& \simeq \tilde{h}_{+}\left(q_{\max }^{2}\right) \simeq \tilde{h}_{\perp}\left(q_{\max }^{2}\right),
\end{aligned}
$$

and

$$
\begin{aligned}
f_{+}\left(q_{\max }^{2}\right) & \simeq g_{0}\left(q_{\max }^{2}\right) \simeq f_{\perp}\left(q_{\max }^{2}\right) \\
& \simeq h_{+}\left(q_{\max }^{2}\right) \simeq h_{\perp}\left(q_{\max }^{2}\right) .
\end{aligned}
$$

All of the form factors can be written as linear combinations of two Isgur-Wise (IW) functions [26]. These relations are valid in the limit of heavy $b$-quark mass $\left(M_{b} \rightarrow \infty\right)$. In principle, there could be non-negligible perturbative and nonperturbative corrections to these relations. As will be discussed later, we have added a correction factor $(\approx 20 \%)$ to all of these relations.

In the SCET, all of the form factors are approximately equal to a single IW function on the other corner of the phase space, i.e., in the large-recoil limit $\left(q^{2}=0\right)$ [15]:

$$
\begin{aligned}
f_{0}(0) & \simeq g_{+}(0) \simeq g_{\perp}(0) \simeq \tilde{h}_{+}(0) \simeq \tilde{h}_{\perp}(0) \simeq f_{+}(0) \\
& \simeq g_{0}(0) \simeq f_{\perp}(0) \simeq h_{+}(0) \simeq h_{\perp}(0) .
\end{aligned}
$$

These relations are exact only in the limit of large recoil energy, and they receive corrections from perturbative and nonperturbative dynamics. One type of such corrections was estimated in Ref. [15], which is of order $\approx 1 \%$. However, we have been conservative and assumed a correction of $\approx 10 \%$ in all of these relations. More details are discussed later.

We first fit the parameters of the form factors described by Eq. (3), while considering the inputs from lattice QCD and the available experimental data. We do not consider the limits from HQET and SCET as inputs in this part of the analysis. Rather, we check whether or not the extracted $q^{2}$ distributions of form factors satisfy the approximate relations given in HQET and SCET at both of the end points. Finally, we repeat the fit with these inputs as additional constraints, and compare the results from both fits. As will be described later, we add parameters to quantify the discrepancies in the approximate relations between the form factors in HQET and SCET in our analysis. This is to take care of the possible large contributions coming from the missing higher-order pieces in those relations. 


\section{Angular observables}

Expanding the sum in Eq. (1), the decay distribution can be expressed in terms of 34 angular observables as follows [12]:

$$
\begin{aligned}
\frac{\mathrm{d}^{6} \Gamma}{\mathrm{d} q^{2} \mathrm{~d} \vec{\Omega}}= & \frac{3}{32 \pi^{2}}\left(\sum_{i=0}^{34} K_{i}\left(q^{2}\right) f_{i}(\vec{\Omega})\right) \\
\frac{\mathrm{d}^{6} \Gamma}{\mathrm{d} q^{2} \mathrm{~d} \vec{\Omega}}= & \frac{3}{32 \pi^{2}}\left(\left(K_{1} \sin ^{2} \theta_{l}+K_{2} \cos ^{2} \theta_{l}+K_{3} \cos \theta_{l}\right)+\left(K_{4} \sin ^{2} \theta_{l}+K_{5} \cos ^{2} \theta_{l}+K_{6} \cos \theta_{l}\right) \cos \theta_{b}\right. \\
& +\left(K_{7} \sin \theta_{l} \cos \theta_{l}+K_{8} \sin \theta_{l}\right) \sin \theta_{b} \cos \left(\phi_{b}+\phi_{l}\right)+\left(K_{9} \sin \theta_{l} \cos \theta_{l}+K_{10} \sin \theta_{l}\right) \sin \theta_{b} \sin \left(\phi_{b}+\phi_{l}\right) \\
& +\left(K_{11} \sin ^{2} \theta_{l}+K_{12} \cos ^{2} \theta_{l}+K_{13} \cos \theta_{l}\right) \cos \theta+\left(K_{14} \sin ^{2} \theta_{l}+K_{15} \cos ^{2} \theta_{l}+K_{16} \cos \theta_{l}\right) \cos \theta_{b} \cos \theta \\
& +\left(K_{17} \sin \theta_{l} \cos \theta_{l}+K_{18} \sin \theta_{l}\right) \sin \theta_{b} \cos \left(\phi_{b}+\phi_{l}\right) \cos \theta+\left(K_{19} \sin \theta_{l} \cos \theta_{l}+K_{20} \sin \theta_{l}\right) \sin \theta_{b} \sin \left(\phi_{b}+\phi_{l}\right) \cos \theta \\
& +\left(K_{21} \cos \theta_{l} \sin \theta_{l}+K_{22} \sin \theta_{l}\right) \sin \phi_{l} \sin \theta+\left(K_{23} \cos \theta_{l} \sin \theta_{l}+K_{24} \sin \theta_{l}\right) \cos \phi_{l} \sin \theta \\
& +\left(K_{25} \cos \theta_{l} \sin \theta_{l}+K_{26} \sin \theta_{l}\right) \sin \phi_{l} \cos \theta_{b} \sin \theta+\left(K_{27} \cos \theta_{l} \sin \theta_{l}+K_{28} \sin \theta_{l}\right) \cos \phi_{l} \cos \theta_{b} \sin \theta \\
& +\left(K_{29} \cos \theta_{l}+K_{30} \sin ^{2} \theta_{l}\right) \sin \theta_{b} \sin \phi_{b} \sin \theta+\left(K_{31} \cos ^{2} \theta_{l}+K_{32} \sin \theta_{l}\right) \sin \theta_{b} \cos \phi_{b} \sin \theta \\
& \left.+\left(K_{33} \sin ^{2} \theta_{l}\right) \sin \theta_{b} \cos \left(2 \phi_{l}+\phi_{b}\right) \sin \theta+\left(K_{34} \sin ^{2} \theta_{l}\right) \sin \theta_{b} \sin \left(2 \phi_{l}+\phi_{b}\right) \sin \theta\right)
\end{aligned}
$$

Integrating Eq. (9) over $\vec{\Omega}$ yields the differential decay rate as a function of $q^{2}$,

$$
\frac{\mathrm{d} \Gamma}{\mathrm{d} q^{2}}=2 K_{1}+K_{2}
$$

This can be used to define a set of normalized angular observables

$$
\hat{K}_{i}=\frac{K_{i}}{2 K_{1}+K_{2}} .
$$

The first ten angular observables defined in Eq. (9) will survive even if the $\Lambda_{b}$ baryon is unpolarized $\left(P_{\Lambda_{b}}=0\right)$. These are listed in Eq. (B1) in Appendix B. The remaining 24 observables are only nonvanishing if $P_{\Lambda_{b}}$ is nonzero [listed in Eqs. (B1) and (B2)]. Of these, the observables $K_{17}$ through $K_{34}$ also involve new combinations of amplitudes that are not accessible if the $\Lambda_{\mathrm{b}}$ baryon is unpolarized. In the massless-lepton limit, $K_{29}$ and $K_{31}$ are zero. As the imaginary parts of the transversity amplitudes are essentially zero in the SM, the observables $K_{19}, K_{20}, K_{21}, K_{22}, K_{25}, K_{26}, K_{30}$, and $K_{34}$ are consistent with zero in the SM. The observables $K_{29}$ and $K_{31}$ have the prefactor $\left(1-\beta_{l}^{2}\right)$, where $\beta_{l}=\sqrt{1-\left(4 m_{l}^{2}\right) /\left(q^{2}\right)}$ and $m_{l}$ is the mass of the leptons in the final state. For $l=\mu$ and $e$, these prefactors are negligibly small and $K_{29}$ and $K_{31}$ will be insensitive to the fit in such cases.

With the combination of the above-mentioned normalized observables [Eq. (11)], the fraction of longitudinally polarized dileptons $\left(f_{L}\right)$ and the hadron-side forwardbackward asymmetry $\left(A_{\mathrm{FB}}^{h}\right)$ are defined as

$$
\begin{gathered}
f_{L}=2 \hat{K}_{1}-\hat{K}_{2}, \\
A_{\mathrm{FB}}^{h}=\hat{K}_{4}+\frac{1}{2} \hat{K}_{5} .
\end{gathered}
$$

In Fig. 1 we show the sensitivity of different angular observables to $P_{\Lambda_{\mathrm{b}}}$. Using the lattice result [17] of the formfactor parameters up to first order in the polynomial expansion $(N=1)$, we plot the theoretical predictions alongside the experimental results for the angular observables $K_{i}(i=11$ to 34$)$ and we vary $P_{\Lambda_{\mathrm{b}}}$ from -1 to 1 . This shows that, except for observables proportional to imaginary parts of combinations of transversity amplitudes (as explained in the previous paragraph), these vary over a considerably large range with varying $P_{\Lambda_{b}}$. This clearly indicates the importance of a data-driven simultaneous estimation of $P_{\Lambda_{\mathrm{b}}}$ along with the form-factor parameters. As the uncertainty in $P_{\Lambda_{\mathrm{b}}}$ already affects the systematic uncertainties of the observables, we do not expect a precise determination of $P_{\Lambda_{\mathrm{b}}}$, but it is interesting to study the effect of the correlations on the other form-factor parameters.

\section{EXPERIMENTAL INPUTS}

After the first observation of $\Lambda_{b} \rightarrow \Lambda \mu^{+} \mu^{-}$by the CDF Collaboration [20], the differential branching fraction of the decay was studied by the LHCb Collaboration with both 1 [21] and $3 \mathrm{fb}^{-1}$ of integrated luminosity [19]. In the latter study, along with the low- $q^{2}\left(0.1-8.0 \mathrm{GeV}^{2}\right)$ and high- $q^{2}$ $\left(15-20 \mathrm{GeV}^{2}\right)$ regions, evidence of the signal was found between the charmonium resonances $\left(11-12.5 \mathrm{GeV}^{2}\right)$. Though the data in the lowest bin $\left(0.1-2.0 \mathrm{GeV}^{2}\right)$ is expected to be large due to the proximity to a photon 


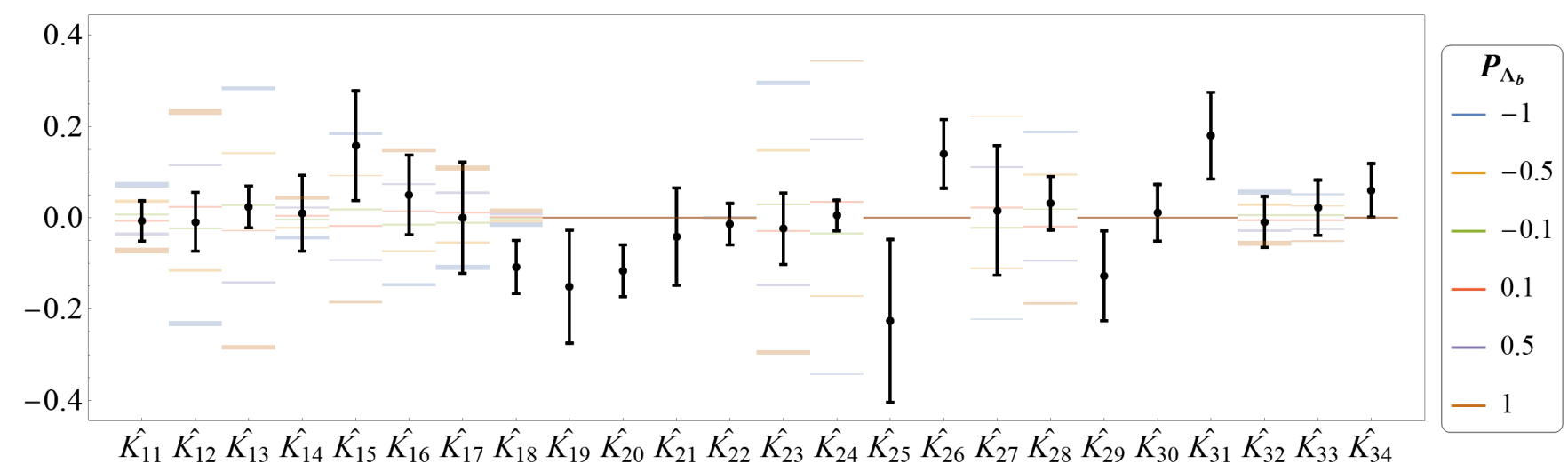

FIG. 1. $\quad P_{\Lambda_{b}}$ dependence of the angular observables. Form-factor parameters are taken from the $N=1$ lattice fit result [17]. The thicknesses of the bands correspond to the respective theoretical uncertainties.

pole, in reality all low- $q^{2}$ data lie lower than the theoretical prediction. We use the differential branching fraction results of these bins in our analysis, and they are listed in Table I.

In addition to these results, Ref. [19] also measured the hadronic angular observables $f_{L}$ and $A_{\mathrm{FB}}^{h}$ in different $q^{2}$ bins, which are listed in Table II and are used in our analysis. Though another set of observables were identified as leptonic forward-backward asymmetries in that paper, an erratum published later showed that these are not the actual $A_{\mathrm{FB}}^{\ell}$ and we refrain from using these in our analysis.

TABLE I. Measured differential branching fraction of $\Lambda_{\mathrm{b}} \rightarrow \Lambda \mu^{+} \mu^{-}$, where the uncertainties are statistical, systematic, and due to the uncertainty on the normalization mode, $\Lambda_{\mathrm{b}} \rightarrow \mathrm{J} / \psi \Lambda$, respectively.

\begin{tabular}{|c|c|}
\hline$q^{2}$ interval $\left[\mathrm{GeV}^{2}\right]$ & $\frac{\mathrm{d} \mathcal{B}}{\mathrm{d} q^{2}} \times 10^{-7}\left[\mathrm{GeV}^{-2}\right]$ \\
\hline $0.1-2.0$ & $0.36_{-0.11-0.02}^{+0.12+0.02} \pm 0.07$ \\
\hline $2.0-4.0$ & $0.11_{-0.09}^{+0.12+0.01} \pm 0.02$ \\
\hline $4.0-6.0$ & $0.02_{-0.00}^{+0.09}{ }_{-0.01}^{+0.01} \pm 0.01$ \\
\hline $6.0-8.0$ & $0.25_{-0.11}^{+0.12}{ }_{-0.01}^{+0.01} \pm 0.05$ \\
\hline $11.0-12.5$ & $0.75_{-0.14}^{+0.15}{ }_{-0.05}^{+0.03} \pm 0.15$ \\
\hline $15.0-16.0$ & $1.12_{-0.18}^{+0.19+0.05} \pm 0.23$ \\
\hline $16.0-18.0$ & $1.22_{-0.14}^{+0.14}{ }_{-0.06}^{+0.03} \pm 0.25$ \\
\hline $18.0-20.0$ & $1.24_{-0.14-0.05}^{+0.14+0.06} \pm 0.26$ \\
\hline
\end{tabular}

TABLE II. Measured values of hadronic angular observables, where the first uncertainties are statistical and the second are systematic.

\begin{tabular}{lcc}
\hline \hline$q^{2}$ interval $\left[\mathrm{GeV}^{2}\right]$ & $f_{\mathrm{L}}$ & $A_{\mathrm{FB}}^{h}$ \\
\hline $0.1-2.0$ & $0.56_{-0.56}^{+0.23} \pm 0.08$ & $-0.12_{-0.28}^{+0.31} \pm 0.15$ \\
$11.0-12.5$ & $0.40_{-0.36}^{+0.36} \pm 0.06$ & $-0.50_{-0.00}^{+0.10} \pm 0.04$ \\
$15.0-16.0$ & $0.49_{-0.30}^{+0.30} \pm 0.05$ & $-0.19_{-0.16}^{+0.14} \pm 0.03$ \\
$16.0-18.0$ & $0.68_{-0.21}^{+0.15} \pm 0.05$ & $-0.44_{-0.05}^{+0.10} \pm 0.03$ \\
$18.0-20.0$ & $0.62_{-0.27}^{+0.24} \pm 0.04$ & $-0.13_{-0.12}^{+0.09} \pm 0.03$ \\
\hline \hline
\end{tabular}

The latest $\mathrm{LHCb}$ measurement [22] with $5 \mathrm{fb}^{-1}$ of integrated luminosity measures all 34 of the angular observables defined in Eq. (9). These are used in our analysis and are listed in Table III. As shown earlier, a couple of other angular observables can be obtained as combinations of these. Of the first ten polarization-independent observables, it has been observed that only $K_{6}$ has a considerable deviation from the SM prediction.

In summary, we start our analysis with a total of 52 observables, some of which may be omitted according to the requirements of the fit, as explained in Sec. IV.

\section{ANALYSIS AND RESULTS}

With the observables listed in Sec. III, we fit the independent form-factor parameters defined in Eq. (3) of Sec. II B, along with the polarization $P_{\Lambda_{\mathrm{b}}}$, in a hybrid of frequentist and Bayesian statistical analyses. ${ }^{2}$

First, we try to fit all 52 of the observables with formfactor parameters up to first order $(N=1)$. These and $P_{\Lambda_{b}}$ constitute a set of 19 parameters. Without any lattice inputs as constraints on the form-factor parameters, i.e., with uniform priors for the parameters between -3 and 3 , the best-fit values are obtained far away from the lattice, but the $p$-value of the resulting fit $(\sim 0.3 \%)$ makes the fit infeasible. Next, we use the $N=1$ fit results from Ref. [17] as lattice inputs and as a multinormal prior. Though the fit results are naturally similar to the lattice ones, no considerable improvement of the fit quality is observed ( $p$-value $\sim 0.5 \%$ ). In the following subsection we describe how to identify influential data, as well as outliers.

\footnotetext{
${ }^{2}$ Parameter estimation is done by populating the posterior parameter space with either uniform or multinormal prior, as the case may be, but the best-fit results (mean) are used to obtain a goodness-of-fit estimate from a $\chi^{2}$ obtained from the corresponding experimental data, the details of which can be found in Appendix C 1.
} 
TABLE III. Angular observables, combining the results of the moments obtained from Run 1 and Run 2 data. The first and second uncertainties are statistical and systematic, respectively.

\begin{tabular}{lrlr}
\hline \hline Obs. & \multicolumn{1}{c}{ Value } & Obs. & Value \\
\hline$K_{1}$ & $0.346 \pm 0.020 \pm 0.004$ & $K_{18}$ & $-0.108 \pm 0.058 \pm 0.008$ \\
$K_{2}$ & $0.308 \pm 0.040 \pm 0.008$ & $K_{19}$ & $-0.151 \pm 0.122 \pm 0.022$ \\
$K_{3}$ & $-0.261 \pm 0.029 \pm 0.006$ & $K_{20}$ & $-0.116 \pm 0.056 \pm 0.008$ \\
$K_{4}$ & $-0.176 \pm 0.046 \pm 0.016$ & $K_{21}$ & $-0.041 \pm 0.105 \pm 0.020$ \\
$K_{5}$ & $-0.251 \pm 0.081 \pm 0.016$ & $K_{22}$ & $-0.014 \pm 0.045 \pm 0.007$ \\
$K_{6}$ & $0.329 \pm 0.055 \pm 0.012$ & $K_{23}$ & $-0.024 \pm 0.077 \pm 0.012$ \\
$K_{7}$ & $-0.015 \pm 0.084 \pm 0.013$ & $K_{24}$ & $0.005 \pm 0.033 \pm 0.005$ \\
$K_{8}$ & $-0.099 \pm 0.037 \pm 0.012$ & $K_{25}$ & $-0.226 \pm 0.176 \pm 0.030$ \\
$K_{9}$ & $0.005 \pm 0.084 \pm 0.012$ & $K_{26}$ & $0.140 \pm 0.074 \pm 0.014$ \\
$K_{10}$ & $-0.045 \pm 0.037 \pm 0.006$ & $K_{27}$ & $0.016 \pm 0.140 \pm 0.025$ \\
$K_{11}$ & $-0.007 \pm 0.043 \pm 0.009$ & $K_{28}$ & $0.032 \pm 0.058 \pm 0.009$ \\
$K_{12}$ & $-0.009 \pm 0.063 \pm 0.014$ & $K_{29}$ & $-0.127 \pm 0.097 \pm 0.016$ \\
$K_{13}$ & $0.024 \pm 0.045 \pm 0.010$ & $K_{30}$ & $0.011 \pm 0.061 \pm 0.011$ \\
$K_{14}$ & $0.010 \pm 0.082 \pm 0.013$ & $K_{31}$ & $0.180 \pm 0.094 \pm 0.015$ \\
$K_{15}$ & $0.158 \pm 0.117 \pm 0.027$ & $K_{32}$ & $-0.009 \pm 0.055 \pm 0.008$ \\
$K_{16}$ & $0.050 \pm 0.084 \pm 0.023$ & $K_{33}$ & $0.022 \pm 0.060 \pm 0.009$ \\
$K_{17}$ & $-0.000 \pm 0.120 \pm 0.022$ & $K_{34}$ & $0.060 \pm 0.058 \pm 0.009$ \\
\hline \hline
\end{tabular}

\section{A. "Influential" data}

The above-mentioned result does not come as a surprise if one checks the relative deviations between the experimental and theoretical estimates of some of the observables. As an example, we can see from Fig. 2 that the LHCb measurement of $K_{6}$ is quite different from its SM estimate. Observables like this are bound to affect and (as a result) worsen the quality of our fits. To illustrate this point and to identify the data points that are outliers as well as influential points, we first define a pull $[27,28]$,

$$
\operatorname{pull}\left(\mathcal{O}_{i}\right)=\left|\frac{\mathcal{O}_{i}^{\exp }-\mathcal{O}_{i}^{\text {fit }}}{\sigma_{i}^{\exp }}\right|,
$$

where $\mathcal{O}_{i}$ is the observable in question, $\mathcal{O}_{i}^{\exp }$ is its experimentally measured value, $\mathcal{O}_{i}^{\text {fit }}$ is its value with the best-fit results of the parameters, and $\sigma^{\exp }$ is the experimental uncertainty of that observable.

Figure 3 shows the distribution of the pull's. It is clear from the figure that the $d \mathcal{B} / d q^{2}$ in the third low- $q^{2}$ bin is the biggest outlier, with a pull $>3$. As being an outlier is not the only criterion to quantify the influence of a datum on a fit, we calculate the Cook's distances of these observables as well ${ }^{3}[30,31]$. By "influential observation" we mean one or several observations whose removal results in a different conclusion in the analysis. Cook's distance is one of many "deletion statistics" used to understand the

${ }^{3}$ For a discussion of Cook's distance and its use in analyses, see Ref. [29].

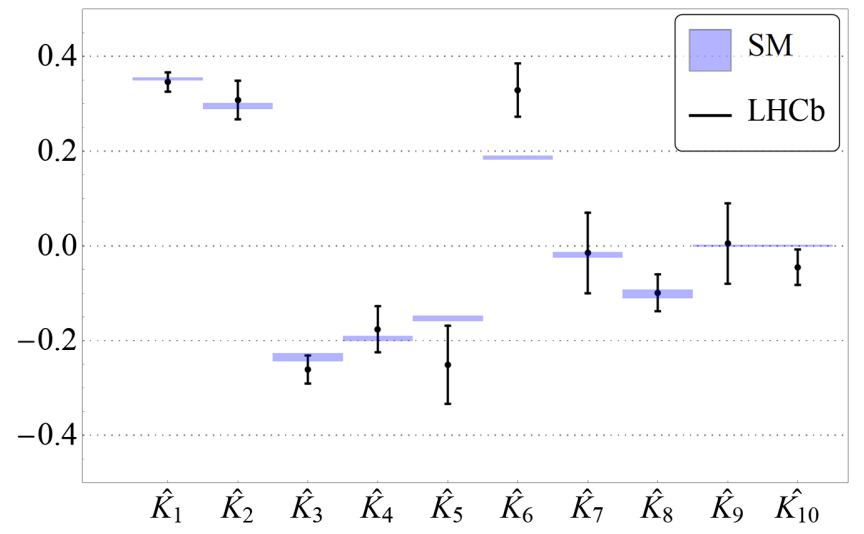

FIG. 2. Comparison of the latest SM estimate (with the $N=2$ result from Ref. [17]) and the experimental result from $\mathrm{LHCb}$ [22] of the polarization-independent angular observables. The thicknesses of the SM bands correspond to the respective theoretical uncertainties.

effect/influence a specific observable has on a fit. It will help us to understand the impact of omitting a case on the estimated regression coefficients. The Cook's distance of the $i$ th observable is

$$
C D_{i}=\frac{\sum_{j=1}^{\mathrm{data}}\left(\hat{y}-\hat{y}_{j(i)}\right)^{2}}{p M S E}
$$

where $\hat{y}$ is the fitted value of the $j$ th observable, $\hat{y}_{j(i)}$ is the same when the $i$ th observable is excluded from the fit, $M S E$ is the mean squared error for the fitted model, and $p$ is the number of regression coefficients. Figure 4 shows the relative sizes of the Cook's distances of the observables. With a Cook cutoff $\sim 0.46$ [32] for the fit, $d \mathcal{B} / d q^{2}(4-6)$ is clearly the most influential point in this fit.

Following the above discussion, we $\operatorname{drop} d \mathcal{B} / d q^{2}(4-6)$ from the fit. As expected, the resulting $p$-value increases by one order (from 0.5 to $5 \%$ ). Still, this is not an acceptable fit and we thus proceed to drop all four outliers $\left[\hat{K}_{6}, \hat{K_{20}}\right.$, $d \mathcal{B} / d q^{2}(4-6)$, and $\left.\mathcal{A}_{\mathrm{FB}}^{\mathrm{bin}}(11.0-12.5)\right]$ from the fit, as shown in Fig. 3. Indeed, this gives a rather good fit ( $p$-value $\sim 68 \%$ ). From hereon, we refer to this fit as N1-Dropl. Recalling the fact that the angular observables only dependent on the imaginary parts of Wilson coefficients are essentially zero in the SM and are insensitive to the parameters (see Appendix B), we also perform a fit by dropping these observables. We refer to this fit as N1Drop 2. ${ }^{4}$ Similar fits are done considering the next higherorder term $(N=2)$ in the $z\left(q^{2}\right)$ expansion of the form factors in Eq. (3). We refer to these fits as N2-Dropl and N2-Drop2, respectively.

\footnotetext{
${ }^{4}$ In general, after dropping the outliers we call these two different types of fits Drop1 and Drop2 scenarios, respectively.
} 


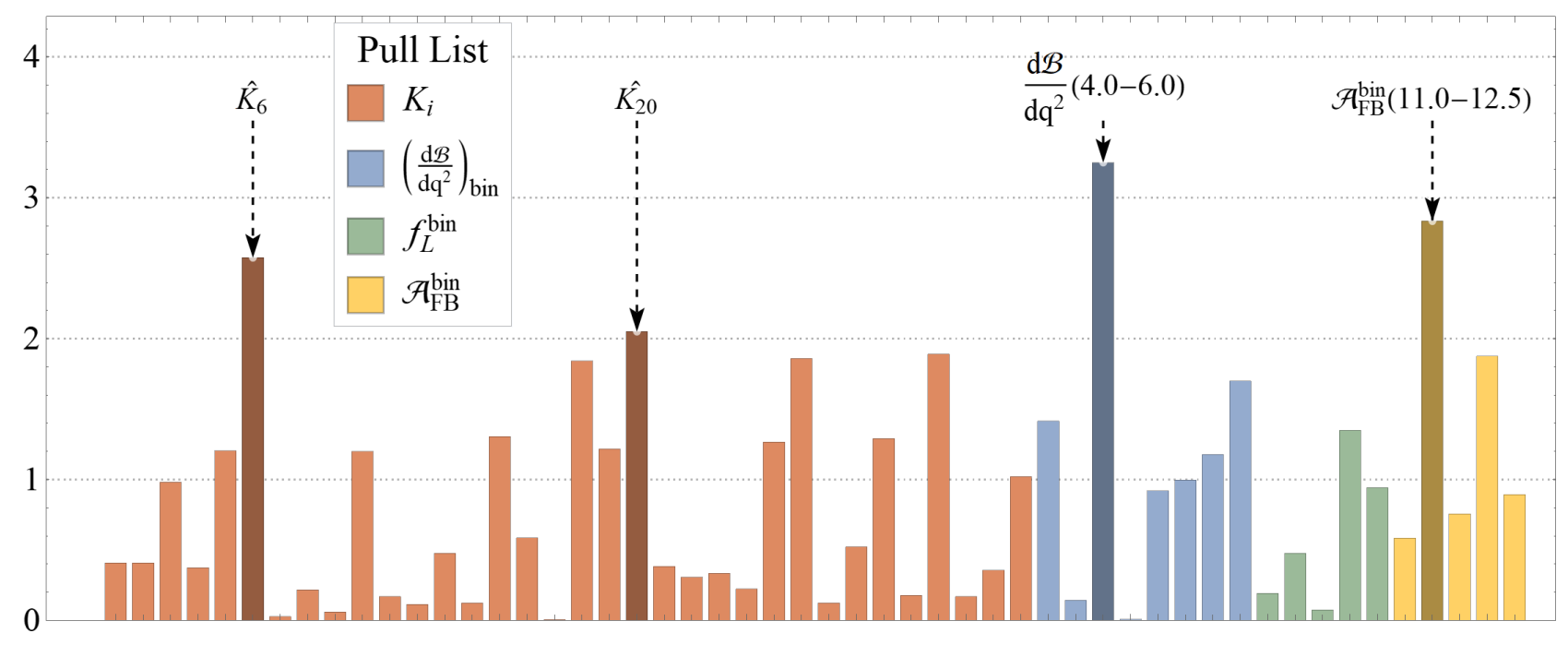

FIG. 3. pull's for all of the observables given with the color code in the index.

\section{B. Different fits}

In this subsection we discuss our different fit procedures, obtained by combining the available inputs in various ways. As mentioned above, the influential data points are dropped in all of these fits. We note from Eq. (3) that the form factors are expanded in different powers of $z\left(q^{2}\right)$. As the variable $z$ is very small, it is natural to expect the terms with higher powers to be insensitive to the fits in general. We perform separate analyses by truncating the series at $N=1$

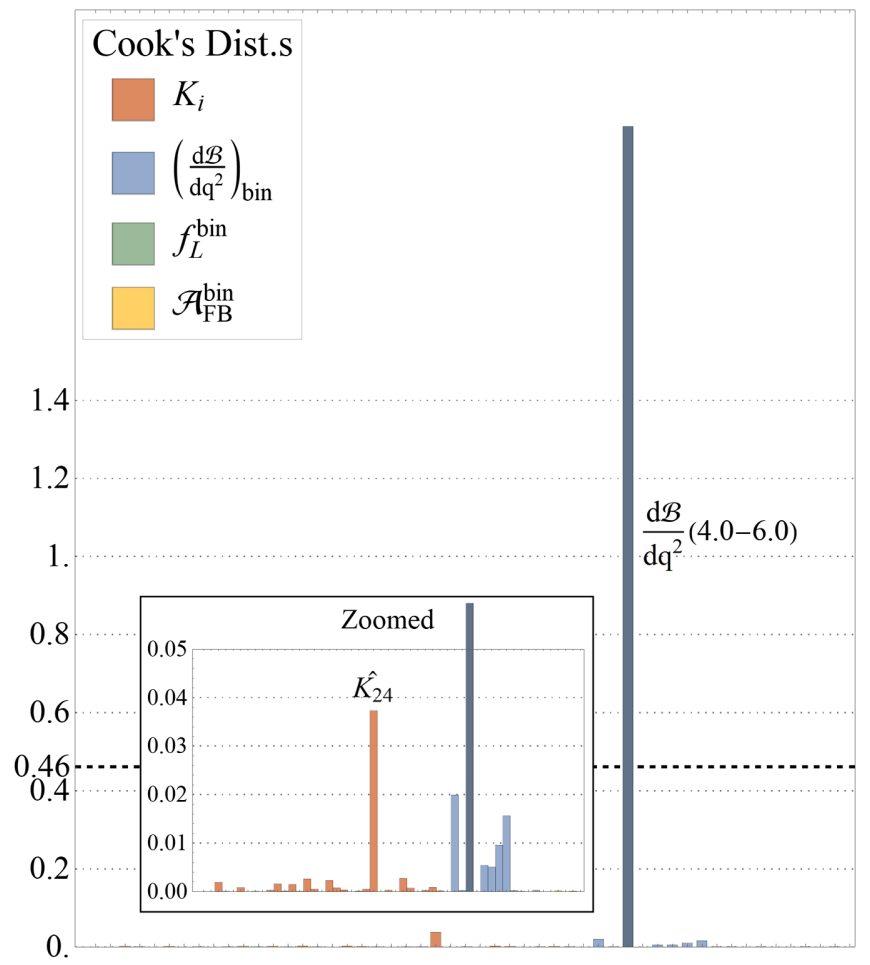

FIG. 4. Cook's distances for all of the observables given with the color code in the index. and $N=2$, respectively. At the moment, it is difficult to analyze the data with higher powers of $N(>2)$.

We have prepared four different data sets in total to understand the trend of the data, the details of which are as follows:

(1) All observables: In this fit we include all of the available experimental inputs. The available lattice inputs from Ref. [17] on the parameters of the form factors for $N=1$ are used as priors in our analysis. Similar sets of fits are repeated for $N=2$, i.e., keeping terms up to $\left[z\left(q^{2}\right)\right]^{2}$ in Eq. (3) [and modifying the constraint equations coming from Eqs. (5c) and (5d) accordingly]. We treat the additional coefficients/parameters at order $N=2$ in the $\left[z\left(q^{2}\right)\right]$ expansion of the form factors as free parameters. We do not use any lattice constraints on these.

(2) Only angular observables: To understand the effects of the angular observables on the parameters of the form factors, we perform another set of fits with only the angular observables. We take the binned data of $\mathcal{A}_{\mathrm{FB}}^{\Lambda}, f_{L}$ [19], and the 34 angular observable from the latest data [22]. In this fit, we do not consider the data on $d \mathcal{B} / d q^{2}$ in different bins as inputs. The methodology of the fits is similar to that in the previous subsection and these too are done for both sets of form-factor parameters, i.e., for $N=1$ and $N=2$. We use a multinormal prior from lattice inputs of the $N=1$ fit in Ref. [17] for all parameters except $P_{\Lambda_{b}}$. We find that this again gives a bad fit ( $p$-value $\sim 9 \%$ ), evidently due to the presence of the observables $\hat{K}_{6}$ and $\hat{K_{20}}$. Dropping those two data points provides a good fit again ( $p$-value $\sim 70 \%$ ). As before, we call these fits as N1-AngDropl and N2-AngDropl, respectively. Here, too, we do not use any lattice inputs for the coefficients at order $N=2$. 
(3) Data-driven fits: We also perform a fit using all of the available experimental data, but without using the lattice inputs as priors. The fit results can be compared with those obtained in the other fits, which might help us to check for any possible tension between the data and the lattice predictions. However, we would like to mention that due to the presence of large inconsistencies between various data points, such fits yield abysmal $p$-values. Also, at the present level of precision, it is hard to analyze these with $N=2$, as in some of the form factors the coefficients of the $\left[z\left(q^{2}\right)\right]^{2}$ term in Eq. (3) are insensitive to the fit. For this reason, we refrain from adding the results of this fit in this paper.

(4) Fits with inputs from SCET and HQET: We repeat all of the above-mentioned fits in the previous three subsections after incorporating the HQET and SCET relations between the form factors at zero and maximum recoil, which are given in Eqs. (6), (7), and (8), respectively. We notice considerable improvements in our data-driven fits because of these inputs. The details of the outcome of this fit are discussed in the next section. The fits with these additional inputs are called NI-Drop1-HS, NI-Drop2-HS, NI-AngDrop1-HS, NIAngDrop2-HS, NI-WOLDropl-HS, and NI-WOLDrop2-HS, respectively (with $I=1$ or 2).

We also check the effect of the nonvanishing lepton mass $\left(m_{\ell}\right)$ in our study. In the limiting case of $m_{\ell} \rightarrow 0$, the two observables $K_{29}$ and $K_{31}$ vanish identically. In addition, a few of the form factors (such as $f_{0}$ and $g_{0}$ ) do not appear in any of the theoretical expressions of the considered observables. While these fits give better $p$-values than N1-Dropl, the parameter spaces are almost identical. Moreover, we choose not to drop estimations of $f_{0}$ and $g_{0}$ for the sake of completeness and we will not discuss the results of these fits.

\section{Outcome of the fits}

The outcome of the fits with all observables and only angular observables are shown in Tables IV and V, respectively. Though the fit results are obtained from a Bayesian analysis, we perform a goodness-of-fit test of the fit using the mean (best-fit) results, and those results are listed in the second and third columns of these tables. The fit results are almost unchanged in the scenarios NI-Drop 1 and NI-Drop2 (with $I=1$ or 2), respectively. However, the fit quality increases considerably in Drop2 scenarios. ${ }^{5}$ Similar to the all-observables scenario, the fit qualities improve after dropping the same set of observables in the fits with only angular observables. Comparing the fits with all observables with those with only angular observables, we notice an overall slight improvement in the $p$-values. Across all of the scenarios, the best-fit values of the

\footnotetext{
${ }^{5}$ Later in our analysis, most of our important results will be presented in Drop2 scenarios.
} 


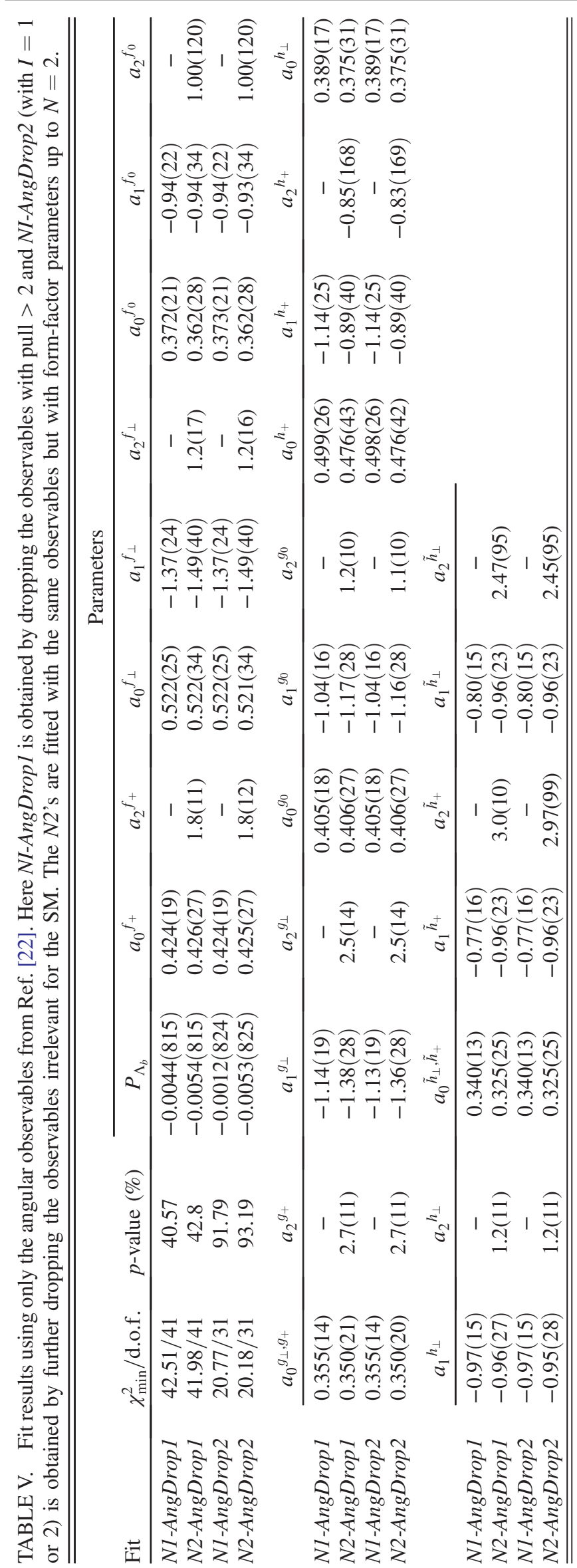

respective parameters are consistent with each other within their respective uncertainties as well. This is due to the dominance of the lattice results (with relatively small uncertainties) in the fits. This is also reflected by the relative invariance of the observable-estimates from two representative scenarios, as shown in Fig. 5.

In Fig. 6 the posterior distributions of the form-factor parameters (up to $N=1$ ) are compared with their respective prior distributions (from Ref. [17]). We do not see any noticeable discrepancies. As the priors are informative here and the data is not precise enough to dominate posterior distributions at the moment, the prior distributions essentially determine the nature of the posteriors (mixtures of prior and likelihood of data). With more precise data, the comparison will be useful to pinpoint any discrepancies between the data and lattice results. We also face our first hurdle in the $N=2$ case for the fits with or without partial lattice constraints. We get completely flat and highly uncertain posteriors for most of the newly introduced higher-order parameters, and consequently the fits do not converge. We surmise that the $N=2$ fits are insensitive to the data and must have lattice constraints, at least at the present experimental precision.

As shown in Eqs. (6), (7), and (8), there are specific relations between the form factors at the zero and maximum recoil angle of the final-state baryon. It is important to check whether the form factors obtained from our fit results satisfy all of these relations. Using different fit results of allobservables fits, we compare the numerical values of the form factors at zero recoil and maximum recoil in Fig. 7. We note that all of the form factors satisfy the relations given in Eqs. (6), (7), and (8), respectively, within their $2 \sigma$ credible intervals (CI). However, there are some discrepancies in some of the relations at their $1 \sigma$ CIs, especially for the fit results with $N=1$. Consequently, the latticepredicted results have some degree of disagreement with the respective SCET and HQET expectations at the end points of $q^{2}$ distributions of the form factors, which are more prominent in the case of HQET (zero recoil).

For all of the cases listed in Tables IV and V, the fits are repeated after incorporating the relations between the form factors in SCET and HQET. We have added 20\% uncertainty in the HQET relations of the form factors at zero recoil, and about $10 \%$ in the case of the SCET relations. The results are summarized in the first and second rows of Table VI, where we present the results only for the cases with $N=2$ (similar results can be obtained for $N=1$ ). While the quality of the fit has diminished in these cases, it still has considerable statistical significance, the reason for which is clear from the discussion in the previous paragraph. The fit results can be compared with the respective results in Tables IV and III. Though we note a slight shift in the best-fit values in some cases, they are consistent with each other within the $1 \sigma$ CIs, which is also evident from Figs. 6 and 8, respectively. In Fig. 6 


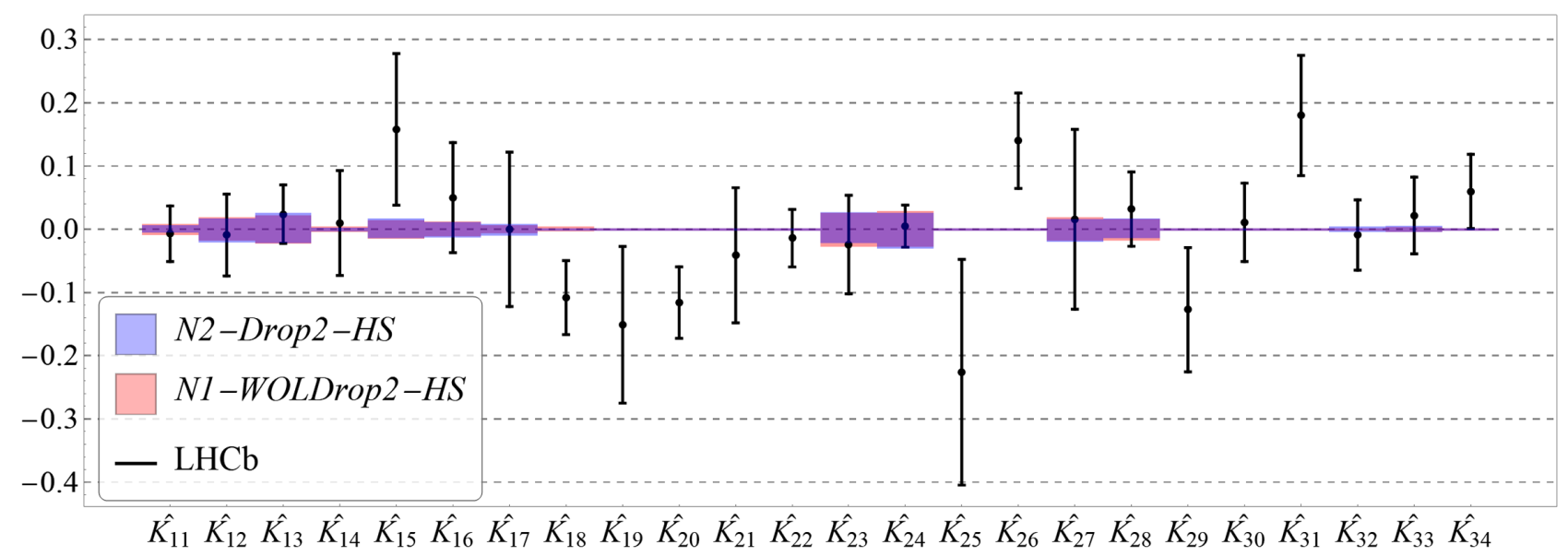

FIG. 5. Comparison of the SM estimate (with the $N=2$ result from the fit dropping the required observables) and the experimental result from $\mathrm{LHCb}$ [22] polarisation dependent angular observables taking $P_{\Lambda_{b}}=1$. The thicknesses of the SM bands correspond to the respective theoretical uncertainties.

we compare the posterior distributions of the zeroth- and first-order form-factor parameters, with and without the inputs from SCET and HQET. Figure 8 summarizes those for the second-order coefficients.

For our data-driven fits, the form factors are truncated at $N=1$. As mentioned earlier, our data does not have enough precision and data alone is not sufficient to extract the coefficients of the form factors for $N=2$ yet. The results of the fit are shown in the third row of Table VI. The best-fit values of the parameters largely deviate from those obtained in the fit with the lattice results as priors. However, the fit values have significant uncertainties. They are consistent with each other within their $3 \sigma$ CIs, which are also shown in Fig. 6 where the posteriors of the relevant parameters are compared. All in all, though the data-driven results are not entirely consistent with the previous ones including lattice inputs, the data does not show any noticeable discrepancy with the lattice results at the moment.

Results of the extracted $q^{2}$ distributions of the form factors for a few fit scenarios, using the different fit results discussed above, are shown in Fig. 9. Note that the $q^{2}$ distributions are fully consistent with each other in the scenarios N1-Drop2, N2-Drop2, and N2-Drop2-HS. All of these scenarios, which we can refer to as our SM-like scenarios, have lattice inputs as priors and the results are similar in all other such fits. However, the $q^{2}$ distributions obtained using the results of the data-driven fit are not fully consistent with each other. For some of the form factors there are discrepancies in the high- $q^{2}$ regions. In particular, there are noticeable discrepancies in $f_{+}, g_{0}$, and $h_{\perp}$ at $1 \sigma$ CI. As we will see below, this could be due to the observed deviations in the measured values of $d \mathcal{B} / d q^{2}$ from their respective SM predictions in the high- $q^{2}$ bins.

Using the form factors extracted in different fits, we predict various related observables and the results are shown in Fig. 10. We note improvements in the uncertainty estimates of the observables after the use of SCET and HQET relations in the fits. The top row shows the predicted values of $d \mathcal{B} / d q^{2}$ in separate bins (top-left panel) and the corresponding $q^{2}$ distributions (top-right panel), compared with their respective measured values. We summarize the important observations below:

(1) The predictions, obtained from the fits in SM-like scenarios, are consistent with each other within uncertainties, which demonstrates the dominance of lattice results over those of the measured values with larger uncertainties.

(2) Apart from the very low bin $\left[0.1 \leq q^{2}\left(\mathrm{GeV}^{2}\right) \leq 2\right]$ and the bins at high $q^{2}\left(15 \leq q^{2}\left[\mathrm{GeV}^{2}\right) \leq 20\right]$, the predictions using the results of the data-driven fit are consistent with those from other fits. ${ }^{6}$

(3) As expected, the data-driven results are consistent with the respective measured values. However, we note some degree of disagreement in the very low- $q^{2}$ bin. In the middle panels we show the predictions for $f_{L}\left(q^{2}\right)$ in different bins (middle-left panel) and the $q^{2}$ distributions (middle-right panel) for different fit scenarios. Note that the predicted results are consistent with each other in all of the scenarios, as well as the respective measurements in all of the $q^{2}$ regions, though the data-driven results have large errors. Similar plots for the forward-backward asymmetry $\left[A_{\mathrm{FB}}\left(q^{2}\right)\right]$ are shown in the bottom panels. Note that, apart from the bin in between $16 \leq q^{2}\left(\mathrm{GeV}^{2}\right) \leq 18$, the extracted values of forward-backward asymmetries in SM-like scenarios and the data-driven fit are consistent with each other. Also, they are consistent with the measured values in the respective bins.

In Table VII we show the predictions for $R_{\Lambda}^{\mu / e}, R_{\Lambda}^{\tau / e}$, and $R_{\Lambda}^{\tau / \mu}$ in different fit scenarios. Compared to $R_{\Lambda}^{\tau / e}$ or $R_{\Lambda}^{\tau / \mu}$, the

\footnotetext{
${ }^{6}$ Note that we have dropped $d \mathcal{B} / d q^{2}(4-6)$ from the fit.
} 

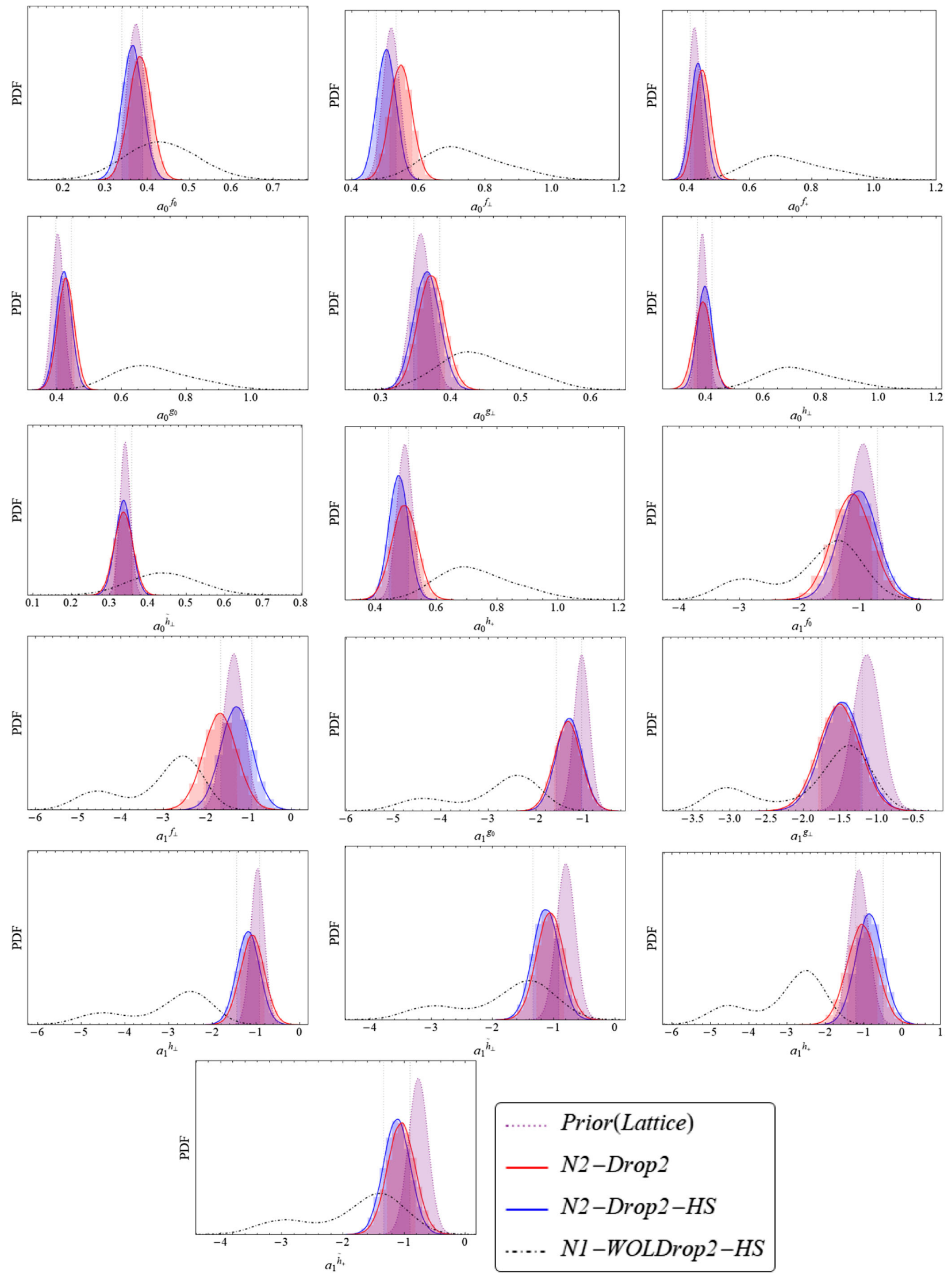

FIG. 6. Comparison of marginal one-dimensional prior and posterior distributions of the " $N=1$ " form-factor parameters with and without lattice inputs. 

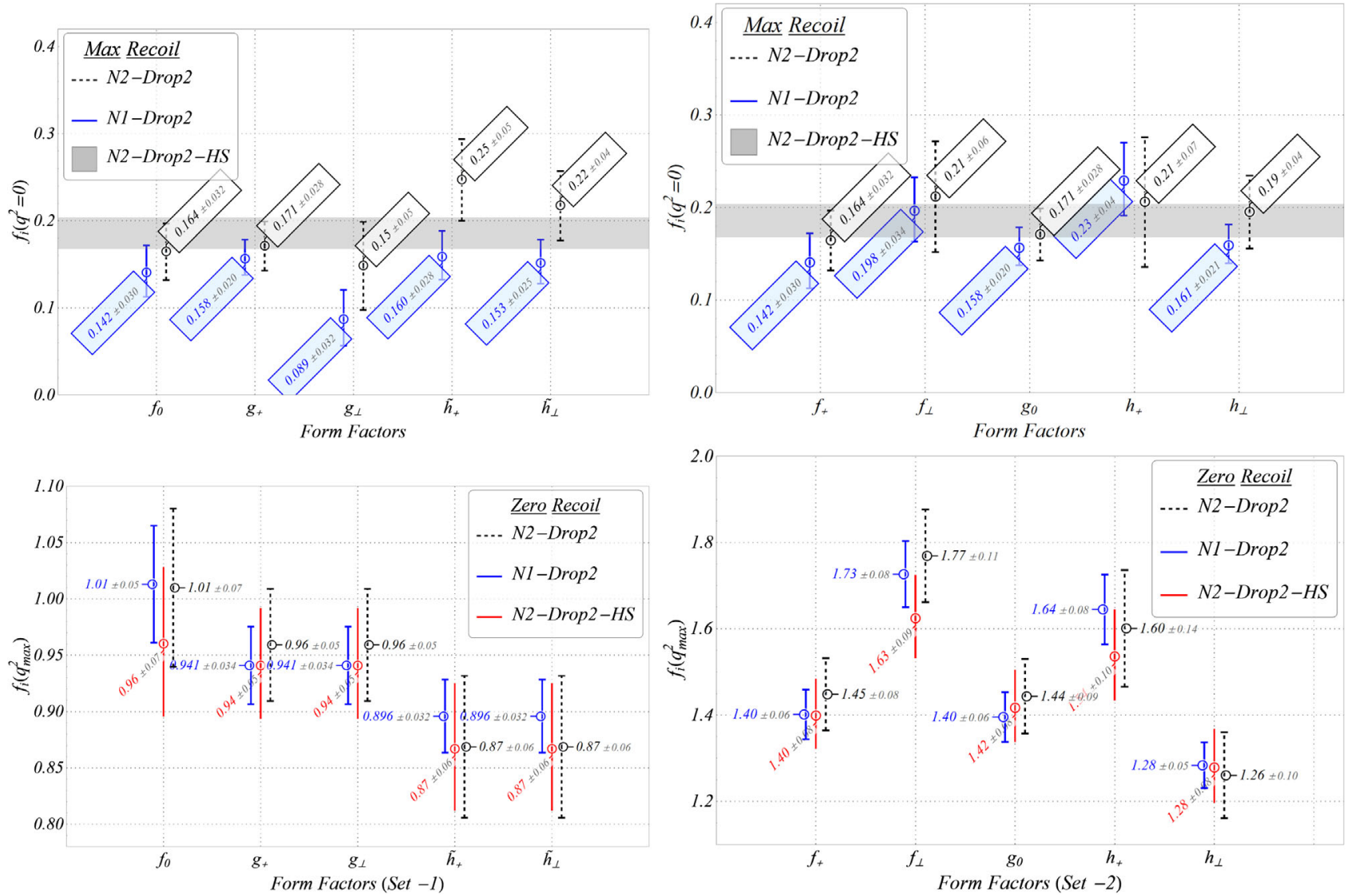

FIG. 7. Form-factor values at zero and max recoil for different fits.
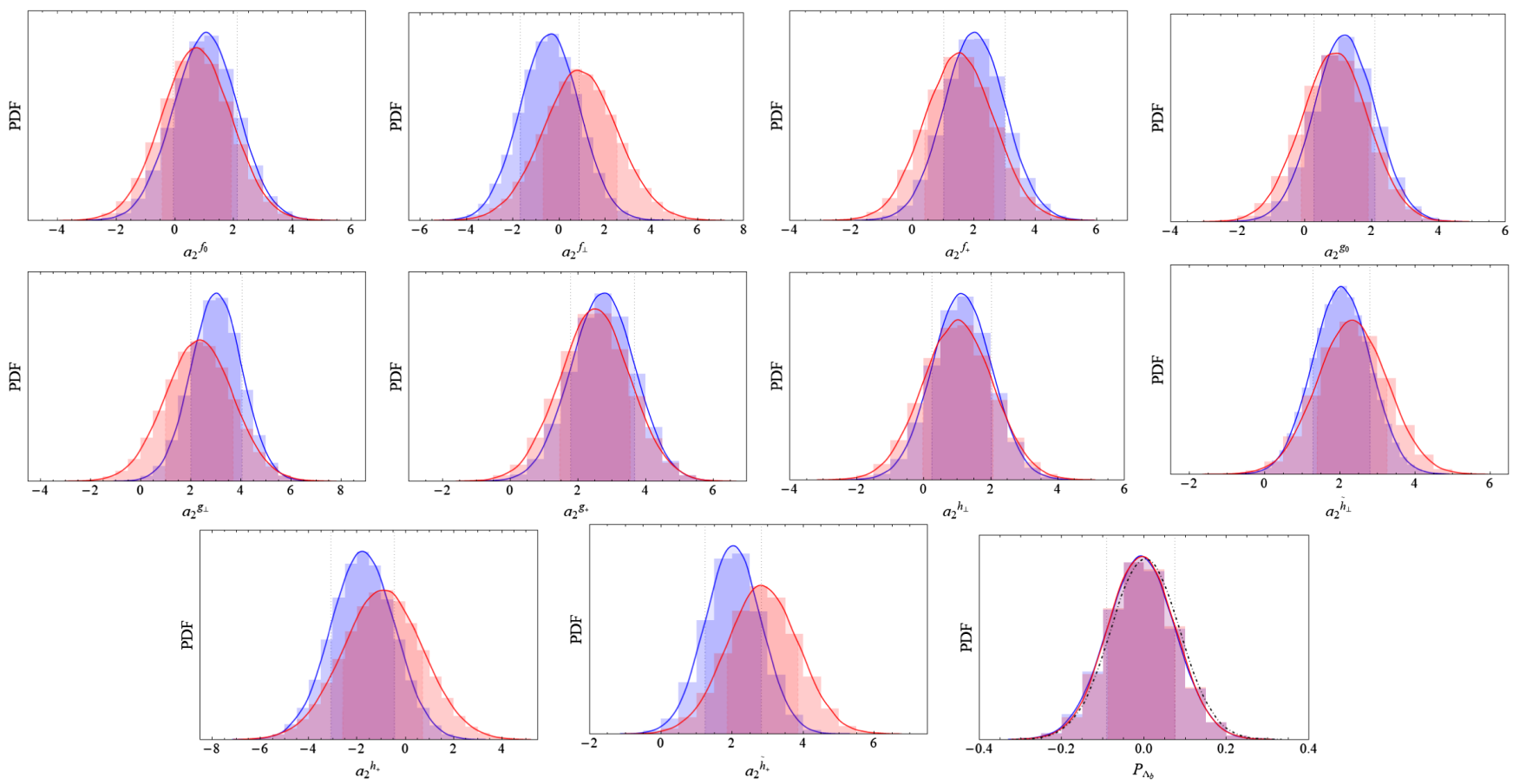

FIG. 8. Marginal one-dimensional posteriors of the coefficients of " $N=2$ " terms of the form factors with (blue) and without (red) the use of HQET and SCET relations [Eqs. (6), (7), and (8)]. 


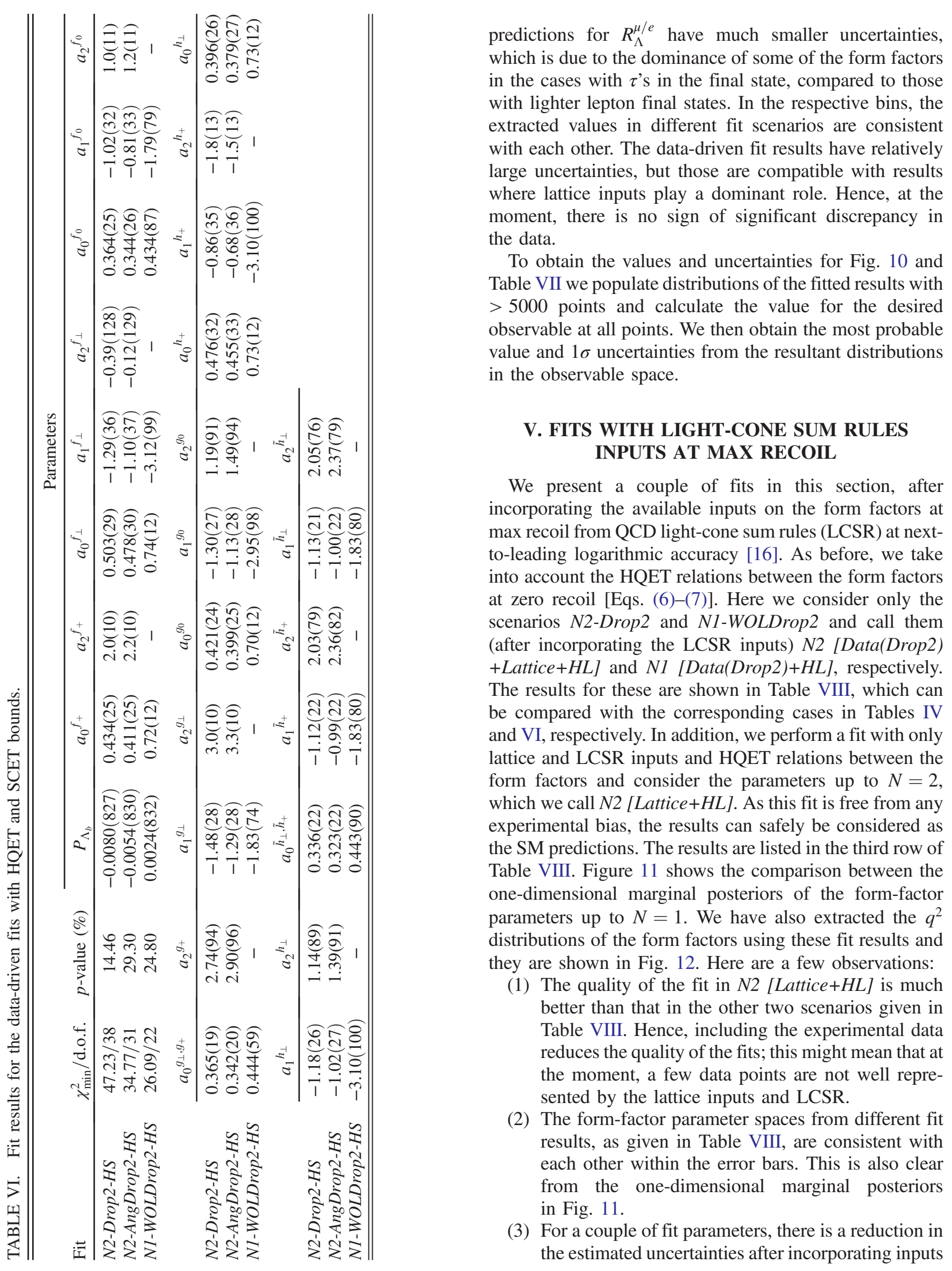



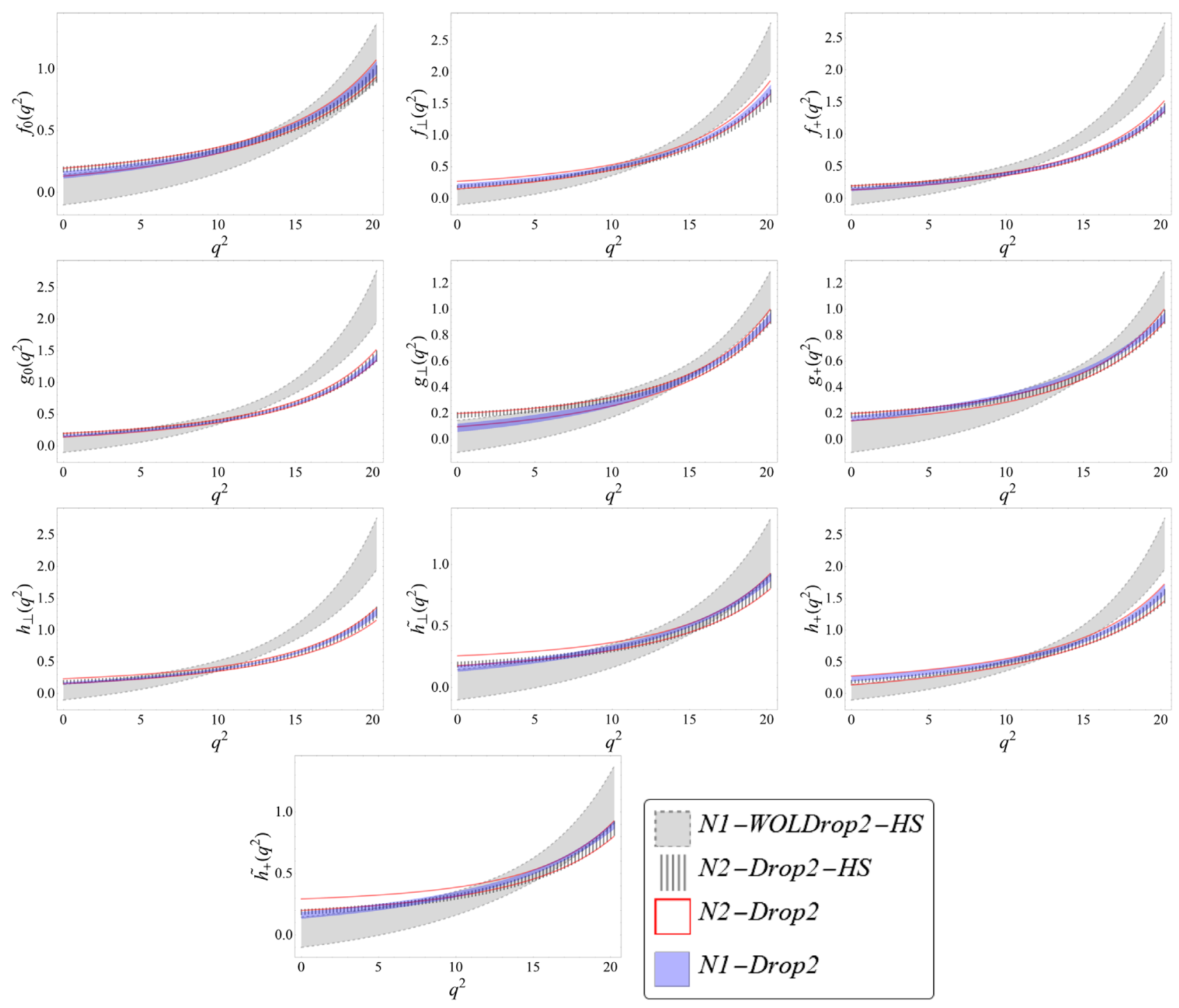

FIG. 9. Form factor distributions in the full $q^{2}$ range with both the data-driven (N1-WOLDropl) and lattice-constrained (N1-Drop1 and N1-Drop2) fits.

from LCSR. A comparison of the scenario N2Drop2 in Table IV with N2 [Data(Drop2)+Lattice $+H L]$ in Table VIII shows that the uncertainties of the coefficients at order $N=0$ and $N=1$ have decreased by $1-2 \%$. However, for some coefficients at order $N=2$, the decrease is as much as $10 \%$. A similar trend is also evident in the scenario N1 [Data (Drop2) +HL] when compared with N1-WOLDrop2 in Table VI. Here, too, we note an improvement of around $10 \%$.

(4) From the $q^{2}$ distributions in Fig. 12, we note that the form factors extracted from N2 [Data(Drop2)+Lattice $+H L]$ and $N 2$ [Lattice $+H L]$ are in good agreement with each other throughout the allowed $q^{2}$ region. However, similar to earlier fits (Fig. 9), the form factors extracted from N1 [Data(Drop2) $+H L$ ] have large errors. In some cases, e.g., for $f_{+}, g_{0}$, and $h_{\perp}$ there are deviations of $>1 \sigma$ in the high- $q^{2}$ regions between the results extracted from $N 2$ $[$ Data(Drop2) + Lattice $+H L]$ and $N 2$ [Lattice $+H L]$.

(5) In scenarios like $N 2[$ Data(Drop2) + Lattice $+H L]$ or $N 2$ [Lattice $+H L]$, the value of the form factors at $q_{\max }^{2}$ remain the same as those obtained earlier in the scenarios N2-Drop2 and N2-Drop2-HS (Fig. 9); this could be due to the dominance of the lattice inputs in the fit. On the contrary, the values of the form factors in the high $q^{2}$ regions decrease in the fit $N 1$ [Data (Drop2) $+H L]$ compared to those obtained in the fit N1-WOLDrop2-HS] [Eq. (9)]. As a result, the differences between the numerical values of the form factors at high- $q^{2}$ decrease.

All of these observations suggest that the lattice results can reproduce LCSR inputs at $q_{\max }^{2}$. However, at the moment, there is a possibility that neither the lattice inputs nor LCSR 

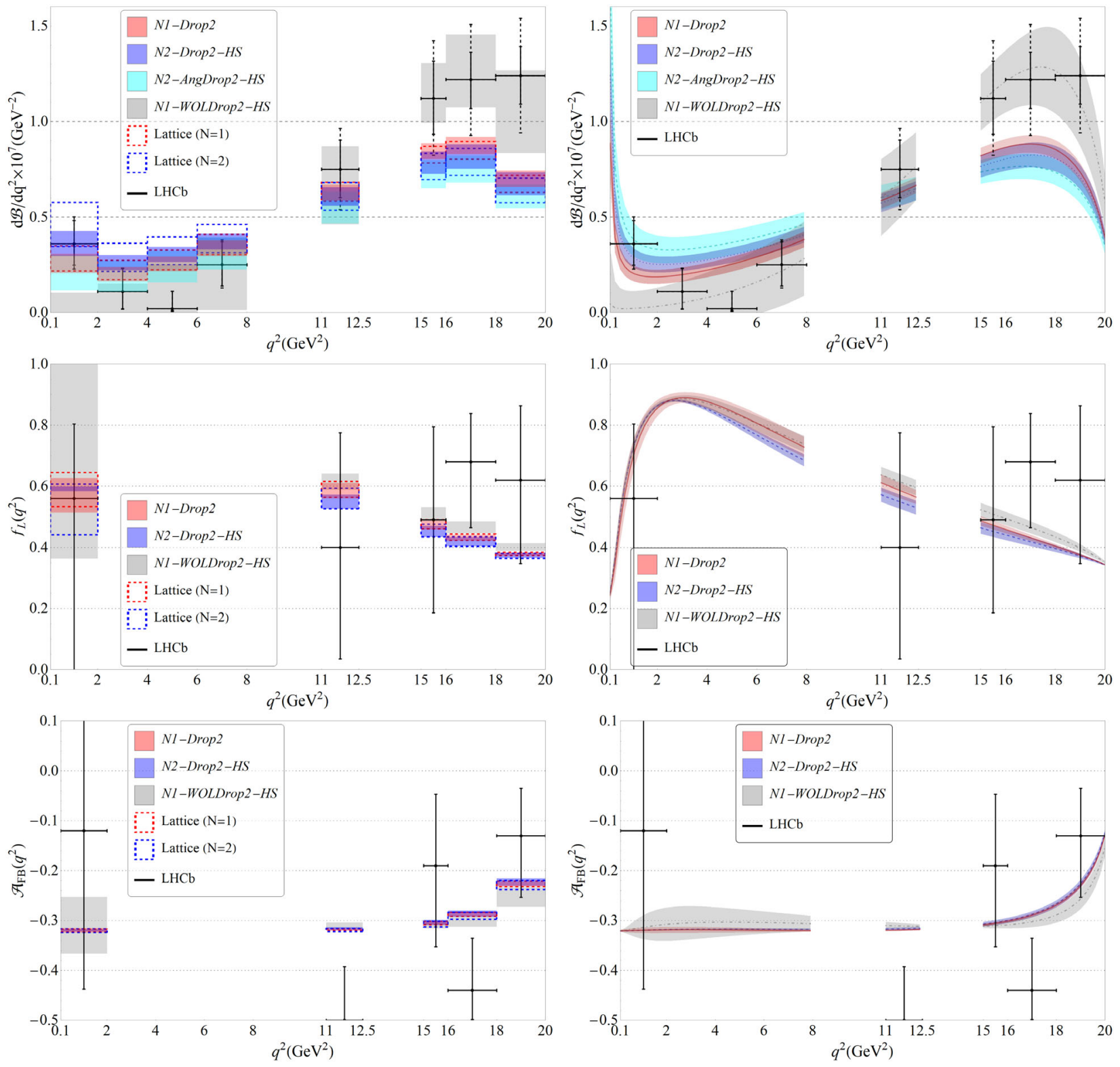

FIG. 10. Comparison of the SM estimate from the fit results (the notations used in the legends are explained in the text) and the lattice results from Ref. [17] with the experimental result from $\mathrm{LHCb}$ [22] of the polarization-independent angular observables. The thicknesses of the bands correspond to the respective theoretical uncertainties.

TABLE VII. $R_{\Lambda}^{\mu / e}$ and $R_{\Lambda}^{\tau / \mu}$ predictions in different bins.

\begin{tabular}{|c|c|c|c|c|c|c|}
\hline \multirow[b]{3}{*}{ Fit } & \multicolumn{4}{|c|}{$R_{\Lambda}^{\mu / e}$} & $R_{\Lambda}^{\tau / e}$ & $R_{\Lambda}^{\tau / \mu}$ \\
\hline & \multicolumn{6}{|c|}{$q^{2}\left(\mathrm{GeV}^{2}\right)$} \\
\hline & $0.1-2$ & $2-8$ & $11-12.5$ & $15-20$ & $15-20$ & $15-20$ \\
\hline N2-Drop2 & $0.976(2)$ & $0.9948(4)$ & $0.99779(8)$ & $0.99838(3)$ & $0.468(5)$ & $0.468(5)$ \\
\hline N2-Drop2-HS & $0.9743(2)$ & $0.9949(1)$ & $0.99781(6)$ & $0.99837(3)$ & $0.466(5)$ & $0.466(4)$ \\
\hline N1-WOLDrop2-HS & $0.974(6)$ & $0.9953(8)$ & $0.99779(9)$ & $0.99835(6)$ & $0.463(10)$ & $0.464(9)$ \\
\hline
\end{tabular}



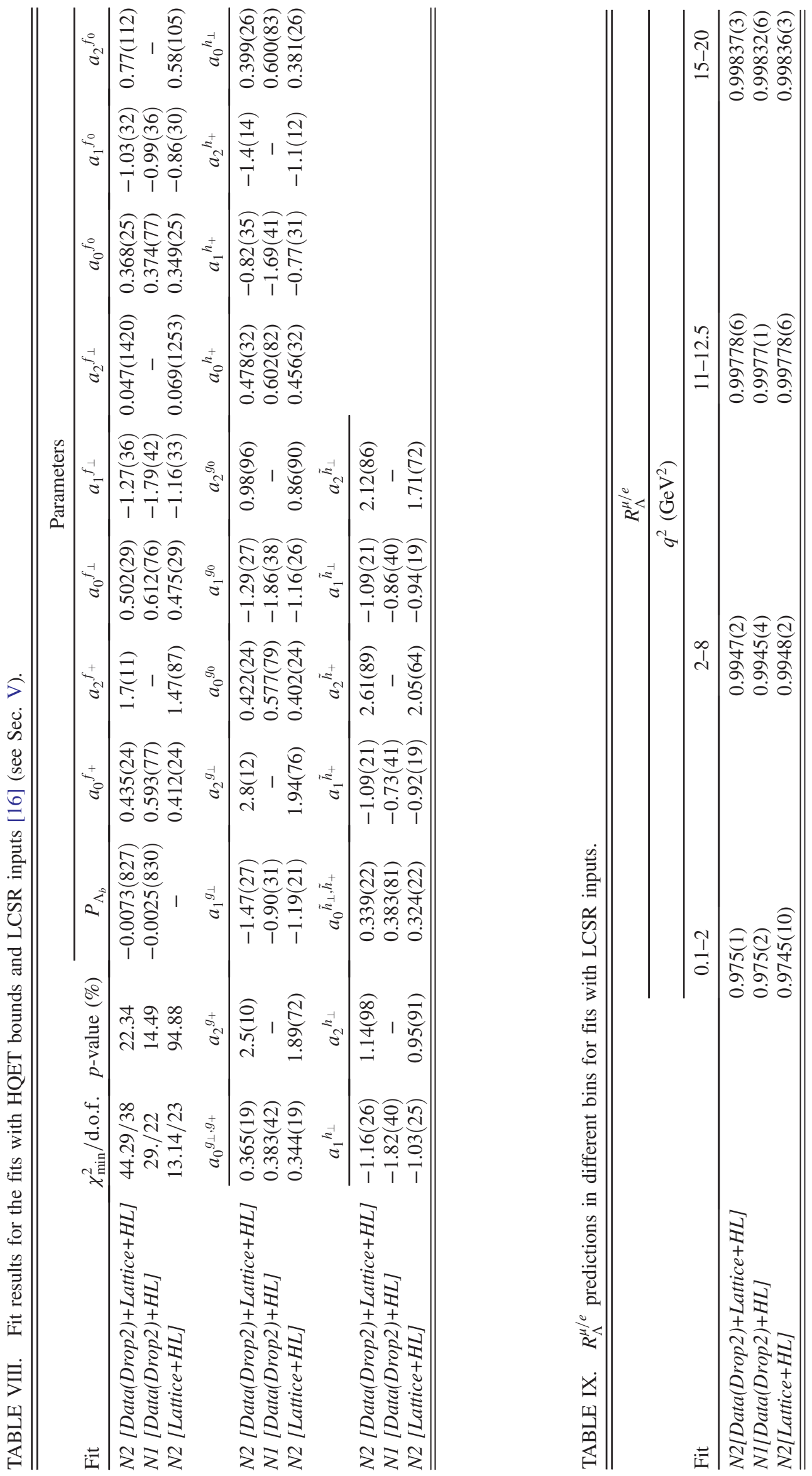

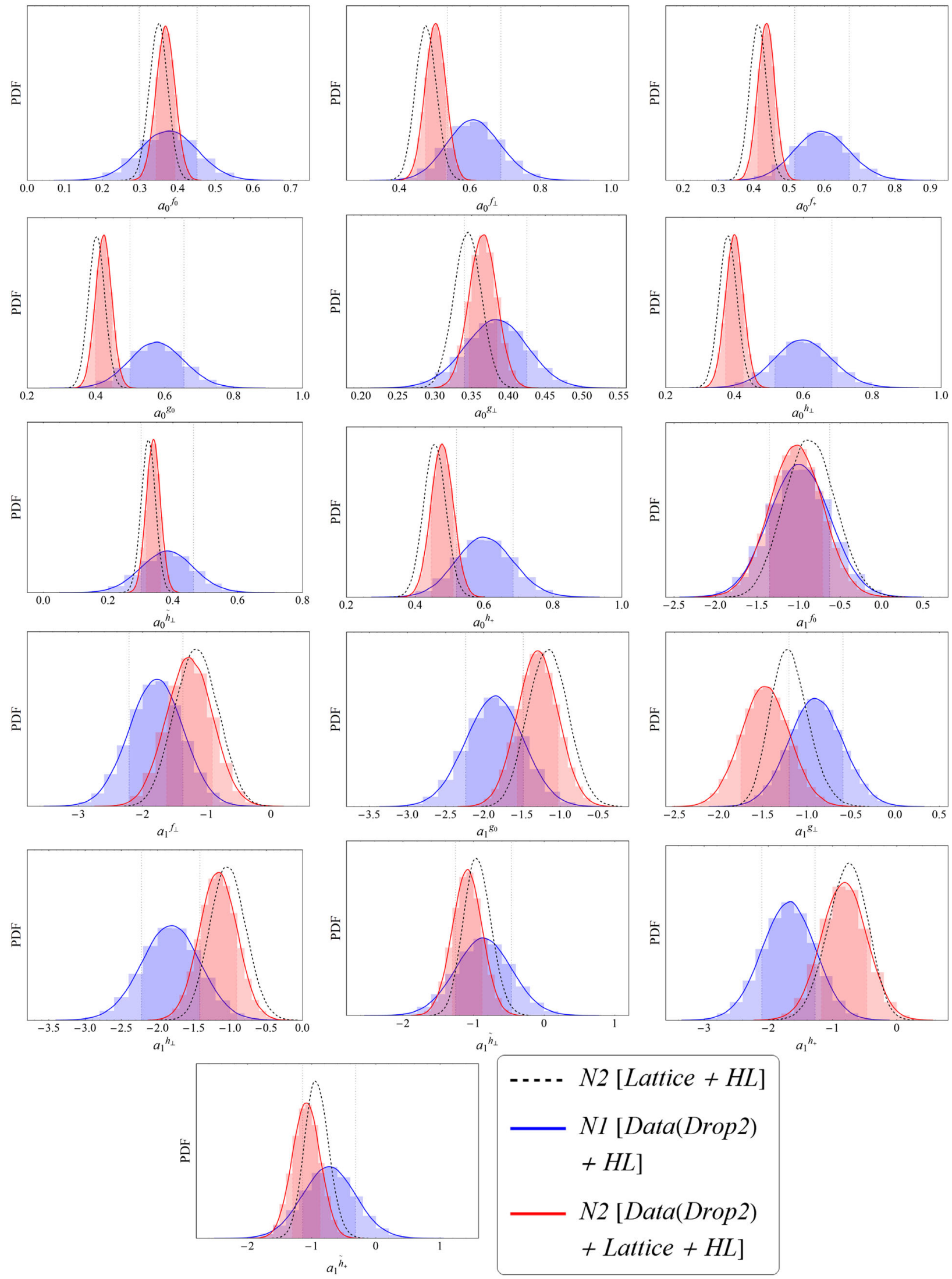

FIG. 11. Comparison of one-dimensional marginal posteriors of the form-factor parameters up to $N=1$, with constraints from HQET and LCSR inputs. 

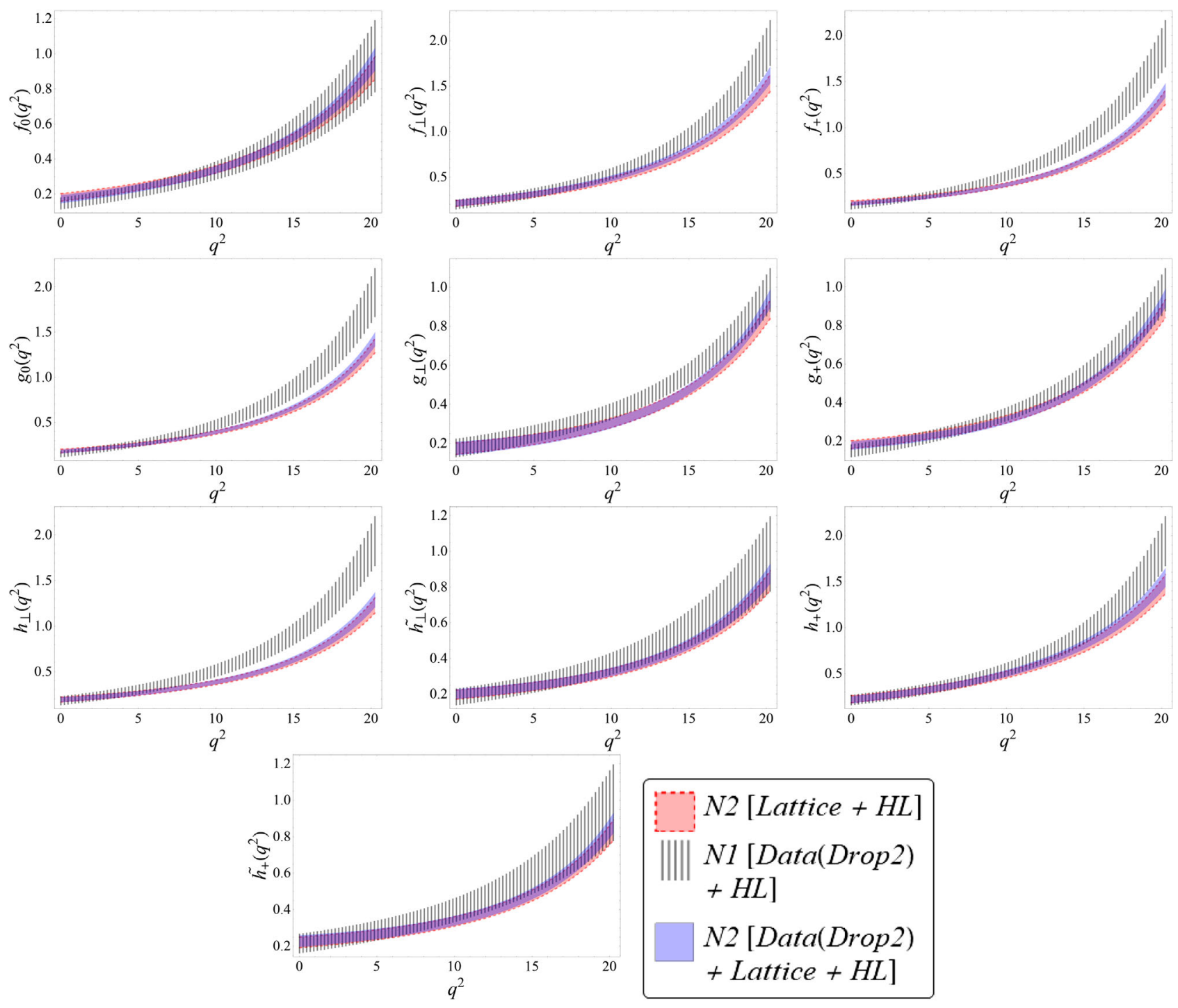

FIG. 12. Form-factor distributions in the full $q^{2}$ range obtained from fits with LCSR constraints.

can accurately represent the experimental data. We need to wait for more precise data to make a conclusion.

In Table IX we show the extracted values of the leptonflavor-universality-violating (LFUV) observable $R_{\Lambda}^{\mu / e}$ in different fit scenarios (as discussed above). In the individual bins, the predicted values are consistent with each other as well as with the predictions obtained earlier in Table VII in the respective bins. The predicted values for $R_{\Lambda}^{\tau / e}$ and $R_{\Lambda}^{\tau / \mu}$ are similar to the one obtained in Table VII, and hence we do not show them explicitly. We have also obtained results similar to Fig. 10 but do not explicitly show them as the results do not change considerably.

\section{SUMMARY}

We have analyzed $\Lambda_{b} \rightarrow \Lambda \ell^{+} \ell^{-}$decays in the framework of the SM with the available data and lattice inputs on the form factors. With the available information, we have defined different fit scenarios. We have tested and utilized the SCET and HQET relations between the form factors at the end points of the $q^{2}$ distributions. From the fit results, we have obtained the $q^{2}$ distributions of the form factors and checked their consistency in different fit scenarios. These form factors were used to predict some observables like $d \mathcal{B} / d q^{2}, f_{L}\left(q^{2}\right), A_{\mathrm{FB}}\left(q^{2}\right)$, and $R_{\Lambda}^{\ell_{i} / \ell_{j}}$. We have pointed out a few influential or problematic data points in a few of the bins, which we have dropped from the fits. A careful examination of these data points is needed from the experimental collaborations.

At the moment, the data has large uncertainties. On the other hand, the lattice results for the form-factor parameters, especially those up to the coefficients of $N=1$ terms in the expansion, have relatively smaller uncertainties. In some of our fits, where we have used the lattice results as priors, the fit results are driven by the lattice inputs and we have identified them as our SM-like results. We have 
noticed that the form factors extracted using these fit results are consistent with the SCET and HQET relations at the end points of the $q^{2}$ distributions. We have also extracted the form factors without any lattice inputs (data-driven fit). If we consider the results at their $1 \sigma$ CIs, a few of them are not consistent with the respective SM-like results in the high- $q^{2}$ regions. However, they are all consistent with the SM-like results at $3 \sigma \mathrm{CI}$. Though a similar conclusion holds for the predictions of $d \mathcal{B} / d q^{2}$, for the cases of $f_{L}\left(q^{2}\right)$ and $A_{\mathrm{FB}}\left(q^{2}\right)$ the respective $q^{2}$ distributions are consistent with each other across all of the fits. The extracted values deviate from their respective measured values in only a few of the bins, which is probably an issue related to the measurement. Finally, our predictions for $R_{\Lambda}^{\mu / e}, R_{\Lambda}^{\tau / e}$, and $R_{\Lambda}^{\tau / \mu}$ from the data-driven fit and the corresponding SM-like predictions are consistent with each other at $1 \sigma$ CI. However, the data-driven results have larger uncertainties compared to their SM-like results.

We have repeated a few of the fits after incorporating the inputs on the form factors at maximum recoil from LCSR at next-to-leading-order accuracy. These fits also include information from lattice and/or experimental data. The additional inputs from LCSR improve the earlier fits of similar type. We noticed a reduction in the estimated uncertainty in some of the fit parameters, which could be as much as $10 \%$. We have also extracted the $q^{2}$ distributions of the form factors and the LFUV observables for all of these scenarios, as defined above. The conclusions are similar, as mentioned above. Among these scenarios, we noted one situation where the inputs from the experimental data were not used, which we called our SM fit. In that fit the predicted values of $R_{\Lambda}^{\mu / e}$ in different bins are consistent with the estimates in other fits in the respective bins.

\section{ACKNOWLEDGMENTS}

This project, S. N., and S. K. P. are supported by the Science and Engineering Research Board, Govt. of India, under the Grant No. CRG/2018/001260.

\section{APPENDIX A: FORM FACTORS}

Here we define the form factors used throughout the paper [15]:

$$
\begin{aligned}
\left\langle\Lambda\left(p^{\prime}, s^{\prime}\right)\left|\bar{s} \gamma^{\mu} b\right| \Lambda_{b}(p, s)\right\rangle= & \bar{u}_{\Lambda}\left(p^{\prime}, s^{\prime}\right)\left[f_{0}\left(q^{2}\right)\left(m_{\Lambda_{b}}-m_{\Lambda}\right) \frac{q^{\mu}}{q^{2}}+f_{+}\left(q^{2}\right) \frac{m_{\Lambda_{b}}+m_{\Lambda}}{s_{+}}\left(p^{\mu}+p^{\prime \mu}-\left(m_{\Lambda_{b}}^{2}-m_{\Lambda}^{2}\right) \frac{q^{\mu}}{q^{2}}\right)\right. \\
+ & \left.f_{\perp}\left(q^{2}\right)\left(\gamma^{\mu}-\frac{2 m_{\Lambda}}{s_{+}} p^{\mu}-\frac{2 m_{\Lambda_{b}}}{s_{+}} p^{\prime \mu}\right)\right] u_{\Lambda_{b}}(p, s) \\
\left\langle\Lambda\left(p^{\prime}, s^{\prime}\right)\left|\bar{s} \gamma^{\mu} \gamma_{5} b\right| \Lambda_{b}(p, s)\right\rangle= & -\bar{u}_{\Lambda}\left(p^{\prime}, s^{\prime}\right) \gamma_{5}\left[g_{0}\left(q^{2}\right)\left(m_{\Lambda_{b}}+m_{\Lambda}\right) \frac{q^{\mu}}{q^{2}}+g_{+}\left(q^{2}\right) \frac{m_{\Lambda_{b}}-m_{\Lambda}}{s_{-}}\left(p^{\mu}+p^{\prime \mu}-\left(m_{\Lambda_{b}}^{2}-m_{\Lambda}^{2}\right) \frac{q^{\mu}}{q^{2}}\right)\right. \\
\left.+g_{\perp}\left(q^{2}\right)\left(\gamma^{\mu}+\frac{2 m_{\Lambda}}{s_{-}} p^{\mu}-\frac{2 m_{\Lambda_{b}}}{s_{-}} p^{\prime \mu}\right)\right] u_{\Lambda_{b}}(p, s), & -\bar{u}_{\Lambda}\left(p^{\prime}, s^{\prime}\right)\left[h_{+}\left(q^{2}\right) \frac{q^{2}}{s_{+}}\left(p^{\mu}+p^{\prime \mu}-\left(m_{\Lambda_{b}}^{2}-m_{\Lambda}^{2}\right) \frac{q^{\mu}}{q^{2}}\right)\right. \\
& \left.+h_{\perp}\left(q^{2}\right)\left(m_{\Lambda_{b}}+m_{\Lambda}\right)\left(\gamma^{\mu}-\frac{2 m_{\Lambda}}{s_{+}} p^{\mu}-\frac{2 m_{\Lambda_{b}}}{s_{+}} p^{\prime \mu}\right)\right] u_{\Lambda_{b}}(p, s), \\
\left\langle\Lambda\left(p^{\prime}, s^{\prime}\right)\left|\bar{s} i \sigma^{\mu \nu} q_{\nu} b\right| \Lambda_{b}(p, s)\right\rangle & -\bar{u}_{\Lambda}\left(p^{\prime}, s^{\prime}\right) \gamma_{5}\left[\tilde{h}_{+}\left(q^{2}\right) \frac{q^{2}}{s_{-}}\left(p^{\mu}+p^{\prime \mu}-\left(m_{\Lambda_{b}}^{2}-m_{\Lambda}^{2}\right) \frac{q^{\mu}}{q^{2}}\right)\right. \\
& \left.+\tilde{h}_{\perp}\left(q^{2}\right)\left(m_{\Lambda_{b}}-m_{\Lambda}\right)\left(\gamma^{\mu}+\frac{2 m_{\Lambda}}{s_{-}} p^{\mu}-\frac{2 m_{\Lambda_{b}}}{s_{-}} p^{\prime \mu}\right)\right] u_{\Lambda_{b}}(p, s),
\end{aligned}
$$

with $q=p-p^{\prime}, \sigma^{\mu \nu}=\frac{i}{2}\left(\gamma^{\mu} \gamma^{\nu}-\gamma^{\nu} \gamma^{\mu}\right)$ and $s_{ \pm}=\left(m_{\Lambda_{b}} \pm m_{\Lambda}\right)^{2}-q^{2}$.

\section{APPENDIX B: ANGULAR OBSERVABLES}

Here we give the full expressions of the angular observables given in Eq. (9) in terms of transversity amplitudes, as in Ref. [12]. Here $\alpha_{\Lambda}$ is the asymmetry parameter of the subsequent decay $\Lambda \rightarrow p \pi$ and its value is $0.642 \pm 0.013[33]:$ 


$$
\begin{aligned}
& K_{1}=\frac{1}{4}\left(\left|A_{\| 1}^{\mathrm{L}}\right|^{2}+\left|A_{\perp 1}^{\mathrm{L}}\right|^{2}+\left|A_{\| 1}^{\mathrm{R}}\right|^{2}+\left|A_{\perp 1}^{\mathrm{R}}\right|^{2}\right)+\frac{1}{4}\left(1+\beta_{l}^{2}\right)\left(\left|A_{\| 0}^{\mathrm{L}}\right|^{2}+\left|A_{\perp 0}^{\mathrm{L}}\right|^{2}+\left|A_{\| 0}^{\mathrm{R}}\right|^{2}+\left|A_{\perp 0}^{\mathrm{R}}\right|^{2}\right) \\
& +\frac{1}{2}\left(1-\beta_{l}^{2}\right) \operatorname{Re}\left(A_{\| 1}^{\mathrm{R}} A_{\| 1}^{* \mathrm{~L}}+A_{\perp 1}^{\mathrm{R}} A_{\perp 1}^{* \mathrm{~L}}+A_{\| 0}^{\mathrm{R}} A_{\| 0}^{* \mathrm{~L}}+A_{\perp 0}^{\mathrm{R}} A_{\perp 0}^{* \mathrm{~L}}\right)+\frac{1}{2}\left(1-\beta_{l}^{2}\right)\left(\left|A_{\| t}\right|^{2}+\left|A_{\perp t}\right|^{2}\right), \\
& K_{2}=\frac{1}{4}\left(1+\beta_{l}^{2}\right)\left(\left|A_{\| 1}^{\mathrm{R}}\right|^{2}+\left|A_{\perp 1}^{\mathrm{R}}\right|^{2}+\left|A_{\| 1}^{\mathrm{L}}\right|^{2}+\left|A_{\perp 1}^{\mathrm{L}}\right|^{2}\right)+\frac{1}{4}\left(1-\beta_{l}^{2}\right)\left(\left|A_{\| 0}^{\mathrm{R}}\right|^{2}+\left|A_{\perp 0}^{\mathrm{R}}\right|^{2}+\left|A_{\| 0}^{\mathrm{L}}\right|^{2}+\left|A_{\perp 0}^{\mathrm{L}}\right|^{2}\right) \\
& +\frac{1}{2}\left(1-\beta_{l}^{2}\right) \operatorname{Re}\left(A_{\| 1}^{\mathrm{R}} A_{\| 1}^{* \mathrm{~L}}+A_{\perp 1}^{\mathrm{R}} A_{\perp 1}^{* \mathrm{~L}}+A_{\| 0}^{\mathrm{R}} A_{\| 0}^{* \mathrm{~L}}+A_{\perp 0}^{\mathrm{R}} A_{\perp 0}^{* \mathrm{~L}}\right)+\frac{1}{2}\left(1-\beta_{l}^{2}\right)\left(\left|A_{\| t}\right|^{2}+\left|A_{\perp t}\right|^{2}\right), \\
& K_{3}=-\beta_{l} \operatorname{Re}\left(A_{\perp 1}^{\mathrm{R}} A_{\| 1}^{* \mathrm{R}}-A_{\perp 1}^{\mathrm{L}} A_{\| 1}^{* \mathrm{~L}}\right), \\
& K_{4}=\frac{1}{2} \alpha_{\Lambda} \operatorname{Re}\left(A_{\perp 1}^{\mathrm{R}} A_{\| 1}^{* \mathrm{R}}+A_{\perp 1}^{\mathrm{L}} A_{\| 1}^{* \mathrm{~L}}\right)+\frac{1}{2} \alpha_{\Lambda}\left(1+\beta_{l}^{2}\right) \operatorname{Re}\left(A_{\perp 0}^{\mathrm{R}} A_{\| 0}^{* \mathrm{R}}+A_{\perp 0}^{\mathrm{L}} A_{\| 0}^{* \mathrm{~L}}\right) \\
& +\frac{1}{2} \alpha_{\Lambda}\left(1-\beta_{l}^{2}\right) \operatorname{Re}\left(A_{\perp 1}^{\mathrm{R}} A_{\| 1}^{* \mathrm{~L}}+A_{\| 1}^{\mathrm{R}} A_{\perp 1}^{* \mathrm{~L}}+A_{\perp 0}^{\mathrm{R}} A_{\| 0}^{* \mathrm{~L}}+A_{\| 0}^{\mathrm{R}} A_{\perp 0}^{* \mathrm{~L}}\right)+\alpha_{\Lambda}\left(1-\beta_{l}^{2}\right) \operatorname{Re}\left(A_{\perp t} A_{\| t}^{*}\right), \\
& K_{5}=\frac{1}{2} \alpha_{\Lambda}\left(1+\beta_{l}^{2}\right) \operatorname{Re}\left(A_{\perp 1}^{\mathrm{R}} A_{\| 1}^{* \mathrm{R}}+A_{\perp 1}^{\mathrm{L}} A_{\| 1}^{* \mathrm{~L}}\right)+\frac{1}{2} \alpha_{\Lambda}\left(1-\beta_{l}^{2}\right) \operatorname{Re}\left(A_{\| 0}^{\mathrm{R}} A_{\perp 0}^{* \mathrm{R}}+A_{\| 0}^{\mathrm{L}} A_{\perp 0}^{* \mathrm{~L}}\right) \\
& +\frac{1}{2} \alpha_{\Lambda}\left(1-\beta_{l}^{2}\right) \operatorname{Re}\left(A_{\perp 1}^{\mathrm{R}} A_{\| 1}^{* \mathrm{~L}}+A_{\| 1}^{\mathrm{R}} A_{\perp 1}^{* \mathrm{~L}}+A_{\perp 0}^{\mathrm{R}} A_{\| 0}^{* \mathrm{~L}}+A_{\| 0}^{\mathrm{R}} A_{\perp 0}^{* \mathrm{~L}}\right)+\alpha_{\Lambda}\left(1-\beta_{l}^{2}\right) \operatorname{Re}\left(A_{\perp t} A_{\| t}^{*}\right), \\
& K_{6}=-\frac{1}{2} \alpha_{\Lambda} \beta_{l}\left(\left|A_{\| 1}^{\mathrm{R}}\right|^{2}+\left|A_{\perp 1}^{\mathrm{R}}\right|^{2}-\left|A_{\| 1}^{\mathrm{L}}\right|^{2}-\left|A_{\perp 1}^{\mathrm{L}}\right|^{2}\right), \\
& K_{7}=\frac{1}{\sqrt{2}} \alpha_{\Lambda} \beta_{l}^{2} \operatorname{Re}\left(A_{\perp 1}^{\mathrm{R}} A_{\| 0}^{* \mathrm{R}}-A_{\| 1}^{\mathrm{R}} A_{\perp 0}^{* \mathrm{R}}+A_{\perp 1}^{\mathrm{L}} A_{\| 0}^{* \mathrm{~L}}-A_{\| 1}^{\mathrm{L}} A_{\perp 0}^{* \mathrm{~L}}\right), \\
& K_{8}=\frac{1}{\sqrt{2}} \alpha_{\Lambda} \beta_{l} \operatorname{Re}\left(A_{\perp 1}^{\mathrm{R}} A_{\perp 0}^{* \mathrm{R}}-A_{\| 1}^{\mathrm{R}} A_{\| 0}^{* \mathrm{R}}-A_{\perp 1}^{\mathrm{L}} A_{\perp 0}^{* \mathrm{~L}}+A_{\| 1}^{\mathrm{L}} A_{\| 0}^{* \mathrm{~L}}\right), \\
& K_{9}=\frac{1}{\sqrt{2}} \alpha_{\Lambda} \beta_{l}^{2} \operatorname{Im}\left(A_{\perp 1}^{R} A_{\perp 0}^{* \mathrm{R}}-A_{\| 1}^{\mathrm{R}} A_{\| 0}^{* \mathrm{R}}+A_{\perp 1}^{\mathrm{L}} A_{\perp 0}^{* \mathrm{~L}}-A_{\| 1}^{\mathrm{L}} A_{\| 0}^{* \mathrm{~L}}\right), \\
& K_{10}=\frac{1}{\sqrt{2}} \alpha_{\Lambda} \beta_{l} \operatorname{Im}\left(A_{\perp 1}^{\mathrm{R}} A_{\| 0}^{* \mathrm{R}}-A_{\| 1}^{\mathrm{R}} A_{\perp 0}^{* \mathrm{R}}-A_{\perp 1}^{\mathrm{L}} A_{\| 0}^{* \mathrm{~L}}+A_{\| 1}^{\mathrm{L}} A_{\perp 0}^{* \mathrm{~L}}\right), \\
& K_{11}=-\frac{1}{2} P_{\Lambda_{\mathrm{b}}} \operatorname{Re}\left(A_{\| 1}^{\mathrm{R}} A_{\perp 1}^{* \mathrm{R}}+A_{\| 1}^{\mathrm{L}} A_{\perp 1}^{* \mathrm{~L}}\right)+\frac{1}{2} P_{\Lambda_{\mathrm{b}}}\left(1+\beta_{l}^{2}\right) \operatorname{Re}\left(A_{\| 0}^{\mathrm{R}} A_{\perp 0}^{* \mathrm{R}}+A_{\| 0}^{\mathrm{L}} A_{\perp 0}^{* \mathrm{~L}}\right) \\
& -\frac{1}{2} P_{\Lambda_{\mathrm{b}}}\left(1-\beta_{l}^{2}\right) \operatorname{Re}\left(A_{\| 1}^{\mathrm{R}} A_{\perp 1}^{* \mathrm{~L}}+A_{\perp 1}^{\mathrm{R}} A_{\| 1}^{* \mathrm{~L}}-A_{\| 0}^{\mathrm{R}} A_{\perp 0}^{* \mathrm{~L}}-A_{\perp 0}^{\mathrm{R}} A_{\| 0}^{* \mathrm{~L}}\right)+P_{\Lambda_{\mathrm{b}}}\left(1-\beta_{l}^{2}\right) \operatorname{Re}\left(A_{\| t} A_{\perp t}^{*}\right), \\
& K_{12}=-\frac{1}{2} P_{\Lambda_{\mathrm{b}}}\left(1+\beta_{l}^{2}\right) \operatorname{Re}\left(A_{\| 1}^{\mathrm{R}} A_{\perp 1}^{* \mathrm{R}}+A_{\| 1}^{\mathrm{L}} A_{\perp 1}^{* \mathrm{~L}}\right)+\frac{1}{2} P_{\Lambda_{\mathrm{b}}}\left(1-\beta_{l}^{2}\right) \operatorname{Re}\left(A_{\| 0}^{\mathrm{R}} A_{\perp 0}^{* \mathrm{R}}+A_{\| 0}^{\mathrm{L}} A_{\perp 0}^{* \mathrm{~L}}\right) \\
& -\frac{1}{2} P_{\Lambda_{\mathrm{b}}}\left(1-\beta_{l}^{2}\right) \operatorname{Re}\left(A_{\| 1}^{\mathrm{R}} A_{\perp 1}^{* \mathrm{~L}}+A_{\perp 1}^{\mathrm{R}} A_{\| 1}^{* \mathrm{~L}}-A_{\| 0}^{\mathrm{R}} A_{\perp 0}^{* \mathrm{~L}}-A_{\perp 0}^{\mathrm{R}} A_{\| 0}^{* \mathrm{~L}}\right)+P_{\Lambda_{\mathrm{b}}}\left(1-\beta_{l}^{2}\right) \operatorname{Re}\left(A_{\| t} A_{\perp t}^{*}\right), \\
& K_{13}=\frac{1}{2} P_{\Lambda_{\mathrm{b}}} \beta_{l}\left(\left|A_{\| 1}^{\mathrm{R}}\right|^{2}+\left|A_{\perp 1}^{\mathrm{R}}\right|^{2}-\left|A_{\| 1}^{\mathrm{L}}\right|^{2}-\left|A_{\perp 1}^{\mathrm{L}}\right|^{2}\right), \\
& K_{14}=-\frac{1}{4} \alpha_{\Lambda} P_{\Lambda_{\mathrm{b}}}\left(\left|A_{\| 1}^{\mathrm{R}}\right|^{2}+\left|A_{\perp 1}^{\mathrm{R}}\right|^{2}+\left|A_{\| 1}^{\mathrm{L}}\right|^{2}+\left|A_{\perp 1}^{\mathrm{L}}\right|^{2}\right)+\frac{1}{4} \alpha_{\Lambda} P_{\Lambda_{\mathrm{b}}}\left(1+\beta_{l}^{2}\right)\left(\left|A_{\| 0}^{\mathrm{R}}\right|^{2}+\left|A_{\perp 0}^{\mathrm{R}}\right|^{2}+\left|A_{\| 0}^{\mathrm{L}}\right|^{2}+\left|A_{\perp 0}^{\mathrm{L}}\right|^{2}\right) \\
& +\frac{1}{2} \alpha_{\Lambda} P_{\Lambda_{\mathrm{b}}}\left(1-\beta_{l}^{2}\right)\left(\left|A_{\| t}\right|^{2}+\left|A_{\perp t}\right|^{2}\right)-\frac{1}{2} \alpha_{\Lambda} P_{\Lambda_{\mathrm{b}}}\left(1-\beta_{l}^{2}\right) \operatorname{Re}\left(A_{\| 1}^{\mathrm{R}} A_{\| 1}^{* \mathrm{~L}}+A_{\perp 1}^{\mathrm{R}} A_{\perp 1}^{* \mathrm{~L}}-A_{\| 0}^{\mathrm{R}} A_{\| 0}^{* \mathrm{~L}}-A_{\perp 0}^{\mathrm{R}} A_{\perp 0}^{* \mathrm{~L}}\right), \\
& K_{15}=-\frac{1}{4} \alpha_{\Lambda} P_{\Lambda_{\mathrm{b}}}\left(1+\beta_{l}^{2}\right)\left(\left|A_{\| 1}^{\mathrm{R}}\right|^{2}+\left|A_{\perp 1}^{\mathrm{R}}\right|^{2}+\left|A_{\| 1}^{\mathrm{L}}\right|^{2}+\left|A_{\perp 1}^{\mathrm{L}}\right|^{2}\right) \frac{1}{4} \alpha_{\Lambda} P_{\Lambda_{\mathrm{b}}}\left(1-\beta_{l}^{2}\right)\left(\left|A_{\| 0}^{\mathrm{R}}\right|^{2}+\left|A_{\perp 0}^{\mathrm{R}}\right|^{2}+\left|A_{\| 0}^{\mathrm{L}}\right|^{2}+\left|A_{\perp 0}^{\mathrm{L}}\right|^{2}\right) \\
& -\frac{1}{2} \alpha_{\Lambda} P_{\Lambda_{\mathrm{b}}}\left(1-\beta_{l}^{2}\right) \operatorname{Re}\left(A_{\| 1}^{\mathrm{R}} A_{\| 1}^{* \mathrm{~L}}+A_{\perp 1}^{\mathrm{R}} A_{\perp 1}^{* \mathrm{~L}}-A_{\| 0}^{\mathrm{R}} A_{\| 0}^{* \mathrm{~L}}-A_{\perp 0}^{\mathrm{R}} A_{\perp 0}^{* \mathrm{~L}}\right)+\frac{1}{2} \alpha_{\Lambda} P_{\Lambda_{\mathrm{b}}}\left(1-\beta_{l}^{2}\right)\left(\left|A_{\| t}\right|^{2}+\left|A_{\perp t}\right|^{2}\right), \\
& K_{16}=\alpha_{\Lambda} P_{\Lambda_{\mathrm{b}}} \beta_{l} \operatorname{Re}\left(A_{\perp 1}^{\mathrm{R}} A_{\| 1}^{* \mathrm{R}}-A_{\perp 1}^{\mathrm{L}} A_{\| 1}^{* \mathrm{~L}}\right) \text {, } \\
& K_{17}=-\frac{1}{\sqrt{2}} \alpha_{\Lambda} P_{\Lambda_{\mathrm{b}}} \beta_{l}^{2} \operatorname{Re}\left(A_{\| 1}^{\mathrm{R}} A_{\| 0}^{* \mathrm{R}}-A_{\perp 1}^{\mathrm{R}} A_{\perp 0}^{* \mathrm{R}}+A_{\| 1}^{\mathrm{L}} A_{\| 0}^{* \mathrm{~L}}-A_{\perp 1}^{\mathrm{L}} A_{\perp 0}^{* \mathrm{~L}}\right),
\end{aligned}
$$




$$
\begin{aligned}
& K_{18}=-\frac{1}{\sqrt{2}} \alpha_{\Lambda} P_{\Lambda_{\mathrm{b}}} \beta_{l} \operatorname{Re}\left(A_{\| 1}^{\mathrm{R}} A_{\perp 0}^{* \mathrm{R}}-A_{\perp 1}^{\mathrm{R}} A_{\| 0}^{* \mathrm{R}}-A_{\| 1}^{\mathrm{L}} A_{\perp 0}^{* \mathrm{~L}}+A_{\perp 1}^{\mathrm{L}} A_{\| 0}^{* \mathrm{~L}}\right), \\
& K_{19}=-\frac{1}{\sqrt{2}} \alpha_{\Lambda} P_{\Lambda_{\mathrm{b}}} \beta_{l}^{2} \operatorname{Im}\left(A_{\| 1}^{\mathrm{R}} A_{\perp 0}^{* \mathrm{R}}-A_{\perp 1}^{\mathrm{R}} A_{\| 0}^{* \mathrm{R}}+A_{\| 1}^{\mathrm{L}} A_{\perp 0}^{* \mathrm{~L}}-A_{\perp 1}^{\mathrm{L}} A_{\| 0}^{* \mathrm{~L}}\right), \\
& K_{20}=-\frac{1}{\sqrt{2}} \alpha_{\Lambda} P_{\Lambda_{\mathrm{b}}} \beta_{l} \operatorname{Im}\left(A_{\| 1}^{\mathrm{R}} A_{\| 0}^{* \mathrm{R}}-A_{\perp 1}^{\mathrm{R}} A_{\perp 0}^{* \mathrm{R}}-A_{\| 1}^{\mathrm{L}} A_{\| 0}^{* \mathrm{~L}}+A_{\perp 1}^{\mathrm{L}} A_{\perp 0}^{* \mathrm{~L}}\right), \\
& K_{21}=\frac{1}{\sqrt{2}} P_{\Lambda_{\mathrm{b}}} \beta_{l}^{2} \operatorname{Im}\left(A_{\| 1}^{\mathrm{R}} A_{\| 0}^{* \mathrm{R}}+A_{\perp 1}^{\mathrm{R}} A_{\perp 0}^{* \mathrm{R}}+A_{\| 1}^{\mathrm{L}} A_{\| 0}^{* \mathrm{~L}}+A_{\perp 1}^{\mathrm{L}} A_{\perp 0}^{* \mathrm{~L}}\right), \\
& K_{22}=-\frac{1}{\sqrt{2}} P_{\Lambda_{\mathrm{b}}} \beta_{l} \operatorname{Im}\left(A_{\| 1}^{\mathrm{R}} A_{\perp 0}^{* \mathrm{R}}+A_{\perp 1}^{\mathrm{R}} A_{\| 0}^{* \mathrm{R}}-A_{\| 1}^{\mathrm{L}} A_{\perp 0}^{* \mathrm{~L}}-A_{\perp 1}^{\mathrm{L}} A_{\| 0}^{* \mathrm{~L}}\right), \\
& K_{23}=-\frac{1}{\sqrt{2}} P_{\Lambda_{\mathrm{b}}} \beta_{l}^{2} \operatorname{Re}\left(A_{\| 1}^{\mathrm{R}} A_{\perp 0}^{* \mathrm{R}}+A_{\perp 1}^{\mathrm{R}} A_{\| 0}^{* \mathrm{R}}+A_{\| 1}^{\mathrm{L}} A_{\perp 0}^{* \mathrm{~L}}+A_{\perp 1}^{\mathrm{L}} A_{\| 0}^{* \mathrm{~L}}\right), \\
& K_{24}=\frac{1}{\sqrt{2}} P_{\Lambda_{\mathrm{b}}} \beta_{l} \operatorname{Re}\left(A_{\| 1}^{\mathrm{R}} A_{\| 0}^{* \mathrm{R}}+A_{\perp 1}^{\mathrm{R}} A_{\perp 0}^{* \mathrm{R}}-A_{\| 1}^{\mathrm{L}} A_{\| 0}^{* \mathrm{~L}}-A_{\perp 1}^{\mathrm{L}} A_{\perp 0}^{* \mathrm{~L}}\right),
\end{aligned}
$$

$$
\begin{aligned}
& K_{25}=\frac{1}{\sqrt{2}} \alpha_{\Lambda} P_{\Lambda_{\mathrm{b}}} \beta_{l}^{2} \operatorname{Im}\left(A_{\| 1}^{\mathrm{R}} A_{\perp 0}^{* \mathrm{R}}+A_{\perp 1}^{\mathrm{R}} A_{\| 0}^{* \mathrm{R}}+A_{\| 1}^{\mathrm{L}} A_{\perp 0}^{* \mathrm{~L}}+A_{\perp 1}^{\mathrm{L}} A_{\| 0}^{* \mathrm{~L}}\right), \\
& K_{26}=-\frac{1}{\sqrt{2}} \alpha_{\Lambda} P_{\Lambda_{\mathrm{b}}} \beta_{l} \operatorname{Im}\left(A_{\| 1}^{\mathrm{R}} A_{\| 0}^{* \mathrm{R}}+A_{\perp 1}^{\mathrm{R}} A_{\perp 0}^{* \mathrm{R}}-A_{\| 1}^{\mathrm{L}} A_{\| 0}^{* \mathrm{~L}}-A_{\perp 1}^{\mathrm{L}} A_{\perp 0}^{* \mathrm{~L}}\right), \\
& K_{27}=-\frac{1}{\sqrt{2}} \alpha_{\Lambda} P_{\Lambda_{\mathrm{b}}} \beta_{l}^{2} \operatorname{Re}\left(A_{\| 1}^{\mathrm{R}} A_{\| 0}^{* \mathrm{R}}+A_{\perp 1}^{\mathrm{R}} A_{\perp 0}^{* \mathrm{R}}+A_{\| 1}^{\mathrm{L}} A_{\| 0}^{* \mathrm{~L}}+A_{\perp 1}^{\mathrm{L}} A_{\perp 0}^{* \mathrm{~L}}\right), \\
& K_{28}=\frac{1}{\sqrt{2}} \alpha_{\Lambda} P_{\Lambda_{\mathrm{b}}} \beta_{l} \operatorname{Re}\left(A_{\| 1}^{\mathrm{R}} A_{\perp 0}^{* \mathrm{R}}+A_{\perp 1}^{\mathrm{R}} A_{\| 0}^{* \mathrm{R}}-A_{\| 1}^{\mathrm{L}} A_{\perp 0}^{* \mathrm{~L}}-A_{\perp 1}^{\mathrm{L}} A_{\| 0}^{* \mathrm{~L}}\right), \\
& K_{29}=\frac{1}{2} \alpha_{\Lambda} P_{\Lambda_{\mathrm{b}}}\left(1-\beta_{l}^{2}\right) \operatorname{Im}\left(A_{\perp 0}^{\mathrm{R}} A_{\| 0}^{* \mathrm{R}}+A_{\perp 0}^{\mathrm{L}} A_{\| 0}^{* \mathrm{~L}}+A_{\perp 0}^{\mathrm{R}} A_{\| 0}^{* \mathrm{~L}}-A_{\| 0}^{\mathrm{R}} A_{\perp 0}^{* \mathrm{~L}}\right)+\alpha_{\Lambda} P_{\Lambda_{\mathrm{b}}}\left(1-\beta_{l}^{2}\right) \operatorname{Im}\left(A_{\perp t} A_{\| t}^{*}\right), \\
& K_{30}=\frac{1}{2} \alpha_{\Lambda} P_{\Lambda_{\mathrm{b}}}\left(1+\beta_{l}^{2}\right) \operatorname{Im}\left(A_{\perp 0}^{\mathrm{R}} A_{\| 0}^{* \mathrm{R}}+A_{\perp 0}^{\mathrm{L}} A_{\| 0}^{* \mathrm{~L}}\right)+\frac{1}{2} \alpha_{\Lambda} P_{\Lambda_{\mathrm{b}}}\left(1-\beta_{l}^{2}\right) \operatorname{Im}\left(A_{\perp 0}^{\mathrm{R}} A_{\| 0}^{* \mathrm{~L}}-A_{\| 0}^{\mathrm{R}} A_{\perp 0}^{* \mathrm{~L}}\right) \\
& +\alpha_{\Lambda} P_{\Lambda_{\mathrm{b}}}\left(1-\beta_{l}^{2}\right) \operatorname{Im}\left(A_{\perp t} A_{\| t}^{*}\right) \\
& K_{31}=\frac{1}{4} \alpha_{\Lambda} P_{\Lambda_{\mathrm{b}}}\left(1-\beta_{l}^{2}\right)\left(\left|A_{\perp 0}^{\mathrm{R}}\right|^{2}-\left|A_{\| 0}^{\mathrm{R}}\right|^{2}+\left|A_{\perp 0}^{\mathrm{L}}\right|^{2}-\left|A_{\| 0}^{\mathrm{L}}\right|^{2}\right) \\
& +\frac{1}{2} \alpha_{\Lambda} P_{\Lambda_{\mathrm{b}}}\left(1-\beta_{l}^{2}\right) \operatorname{Re}\left(A_{\perp 0}^{\mathrm{R}} A_{\perp 0}^{* \mathrm{~L}}-A_{\| 0}^{\mathrm{R}} A_{\| 0}^{* \mathrm{~L}}\right)+\frac{1}{2} \alpha_{\Lambda} P_{\Lambda_{\mathrm{b}}}\left(1-\beta_{l}^{2}\right)\left(\left|A_{\perp t}\right|^{2}-\left|A_{\| t}\right|^{2}\right), \\
& K_{32}=\frac{1}{4} \alpha_{\Lambda} P_{\Lambda_{\mathrm{b}}}\left(1+\beta_{l}^{2}\right)\left(\left|A_{\perp 0}^{\mathrm{R}}\right|^{2}+\left|A_{\perp 0}^{\mathrm{L}}\right|^{2}-\left|A_{\| 0}^{\mathrm{R}}\right|^{2}-\left|A_{\| 0}^{\mathrm{L}}\right|^{2}\right) \\
& +\frac{1}{2} \alpha_{\Lambda} P_{\Lambda_{\mathrm{b}}}\left(1-\beta_{l}^{2}\right) \operatorname{Re}\left(A_{\perp 0}^{\mathrm{R}} A_{\perp 0}^{* \mathrm{~L}}-A_{\| 0}^{\mathrm{R}} A_{\| 0}^{* \mathrm{~L}}\right)+\frac{1}{2} \alpha_{\Lambda} P_{\Lambda_{\mathrm{b}}}\left(1-\beta_{l}^{2}\right)\left(\left|A_{\perp t}\right|^{2}-\left|A_{\| t}\right|^{2}\right), \\
& K_{33}=\frac{1}{4} \alpha_{\Lambda} P_{\Lambda_{\mathrm{b}}} \beta_{l}^{2}\left(\left|A_{\perp 1}^{\mathrm{R}}\right|^{2}-\left|A_{\| 1}^{\mathrm{R}}\right|^{2}+\left|A_{\perp 1}^{\mathrm{L}}\right|^{2}-\left|A_{\| 1}^{\mathrm{L}}\right|^{2}\right), \\
& K_{34}=\frac{1}{2} \alpha_{\Lambda} P_{\Lambda_{\mathrm{b}}} \beta_{l}^{2} \operatorname{Im}\left(A_{\perp 1}^{\mathrm{R}} A_{\| 1}^{* \mathrm{R}}+A_{\perp 1}^{\mathrm{L}} A_{\| 1}^{* \mathrm{~L}}\right) \text {. }
\end{aligned}
$$




\section{APPENDIX C: FIT METHODOLOGY}

\section{1. $\chi^{2}$ definition}

Here we consider two different ways to fit the parameters. First, a $\chi^{2}$ statistic is defined by considering each of the form-factor parameters as a free parameter in the following way:

$\chi^{2}=\sum_{i, j=1}^{\text {data }}\left(O_{i}^{\text {exp }}-O_{i}^{\text {th }}\right)\left(V^{\text {stat }}+V^{\text {syst }}\right)_{i j}^{-1}\left(O_{j}^{\text {exp }}-O_{j}^{\text {th }}\right)$.

Here $O_{p}^{\text {th }}$ is the theoretical expression and $O_{p}^{\exp }$ is the central value of the experimental result of the $p$ th observable used in the analysis. $V^{\text {type }}$ is the covariance matrix, where "type" is either statistical or systematic. $O_{p}^{\text {th }}$ are functions of the form-factor parameters.
For the second scenario, all of the form-factor parameters are considered as nuisance parameters and the definition of $\chi^{2}$ [Eq. (C1)] is modified as

$\chi^{2}=\sum_{i, j=1}^{\text {data }}\left(O_{i}^{\text {exp }}-O_{i}^{\text {th }}\right)\left(V^{\text {stat }}+V^{\text {syst }}\right)_{i j}^{-1}\left(O_{j}^{\text {exp }}-O_{j}^{\text {th }}\right)+\chi_{\text {Nuis }}^{2}$.

Here $\chi_{\text {Nuis }}^{2}$ is defined as

$$
\chi_{\text {Nuis }}^{2}=\sum_{i, j=1}^{\text {params }}\left(I_{i}^{P}-v_{i}^{p}\right)\left(V^{\text {Nuis }}\right)_{i j}^{-1}\left(I_{j}^{p}-v_{j}^{p}\right) .
$$

In Eq. (C3), $I_{k}^{P}$ and $v_{k}^{P}$ are the $k$ th input parameters and their values, respectively. In our case, their values are constrained by means of the previous lattice fit results.
[1] R. Aaij et al. (LHCb Collaboration), Phys. Rev. Lett. 113, 151601 (2014).

[2] A. Abdesselam et al. (Belle Collaboration), arXiv: 1904.02440.

[3] S. Bhattacharya, A. Biswas, S. Nandi, and S. K. Patra, Phys. Rev. D 101, 055025 (2020).

[4] G. Buchalla, A. J. Buras, and M. E. Lautenbacher, Rev. Mod. Phys. 68, 1125 (1996).

[5] T. Gutsche, M. A. Ivanov, J. G. Korner, V. E. Lyubovitskij, and P. Santorelli, Phys. Rev. D 87, 074031 (2013).

[6] P. Ber, T. Feldmann, and D. van Dyk, J. High Energy Phys. 01 (2015) 155.

[7] L. Mott and W. Roberts, Int. J. Mod. Phys. A 27, 1250016 (2012).

[8] S. Roy, R. Sain, and R. Sinha, Phys. Rev. D 96, 116005 (2017).

[9] D. Das, J. High Energy Phys. 07 (2018) 063.

[10] T. M. Aliev, A. Ozpineci, M. Savci, and C. Yuce, Phys. Lett. B 542, 229 (2002).

[11] C.-S. Huang and H.-G. Yan, Phys. Rev. D 59, 114022 (1999); 61, 039901(E) (2000).

[12] T. Blake and M. Kreps, J. High Energy Phys. 11 (2017) 138.

[13] Y.-m. Wang, Y. Li, and C.-D. Lu, Eur. Phys. J. C 59, 861 (2009).

[14] T. M. Aliev, K. Azizi, and M. Savci, Phys. Rev. D 81, 056006 (2010).

[15] T. Feldmann and M. W. Y. Yip, Phys. Rev. D 85, 014035 (2012); 86, 079901(E) (2012).

[16] Y.-M. Wang and Y.-L. Shen, J. High Energy Phys. 02 (2016) 179.
[17] W. Detmold and S. Meinel, Phys. Rev. D 93, 074501 (2016).

[18] R. Aaij et al. (LHCb Collaboration), Phys. Lett. B 724, 27 (2013).

[19] R. Aaij et al. (LHCb Collaboration), J. High Energy Phys. 06 (2015) 115; 09 (2018) 145(E).

[20] T. Aaltonen et al. (CDF Collaboration), Phys. Rev. Lett. 107, 201802 (2011).

[21] R. Aaij et al. (LHCb Collaboration), Phys. Lett. B 725, 25 (2013).

[22] R. Aaij et al. (LHCb Collaboration), J. High Energy Phys. 09 (2018) 146.

[23] T. Mannel and Y.-M. Wang, J. High Energy Phys. 12 (2011) 067.

[24] P. Bialas, J. G. Korner, M. Kramer, and K. Zalewski, Z. Phys. C 57, 115 (1993).

[25] A. Kadeer, J. G. Korner, and U. Moosbrugger, Eur. Phys. J. C 59, 27 (2009).

[26] T. Mannel, W. Roberts, and Z. Ryzak, Nucl. Phys. B355, 38 (1991).

[27] E. Gross, CERN Yellow Rep.: Sch. Proc. 4, 165 (2017).

[28] L. Demortier and L. Lyons (CDF Collaboration), Report No. CDF/ANAL/PUBLIC/5776, 2008.

[29] S. Bhattacharya, A. Biswas, Z. Calcuttawala, and S. K. Patra, arXiv:1902.02796.

[30] R. D. Cook, Technometrics 19, 15 (1977).

[31] R. D. Cook, J. Am. Stat. Assoc. 74, 169 (1979).

[32] K. A. Bollen and R. W. Jackman, Sociol. Methods Res. 13, 510 (1985).

[33] K. Olive, Chin. Phys. C 38, 090001 (2014). 